\title{
Fading Multiple Access Relay Channels: Achievable Rates and Opportunistic Scheduling
}

\author{
Lalitha Sankar, Member, IEEE, Yingbin Liang, Member, IEEE, Narayan B. Mandayam, Fellow, IEEE, and \\ H. Vincent Poor, Fellow, IEEE
}

\begin{abstract}
The problem of optimal resource allocation is studied for ergodic fading orthogonal multi-access relay channels (MARCs) in which the users (sources) communicate with a destination with the aid of a half-duplex relay that transmits and receives on orthogonal channels. Under the assumption that the instantaneous fading state information is available at all nodes, the maximum sum-rate and the optimal user and relay power allocations (policies) are developed for a decode-and-forward (DF) relay. A known lemma on the sum-rate of two intersecting polymatroids is used to determine the DF sum-rate and the optimal user and relay policies, and to classify fading MARCs into one of three types: (i) partially clustered MARCs in which a user is clustered either with the relay or with the destination, (ii) clustered MARCs in which all users are either proximal to the relay or to the destination, and (iii) arbitrarily clustered MARCs which are a combination of the first two types. Cutset outer bounds are used to show that DF achieves the capacity region for a sub-class of clustered orthogonal MARCs.
\end{abstract}

Index Terms-Decode-and-forward, ergodic capacity, fading, multiple-access relay channel (MARC), resource allocation.

\section{INTRODUCTION}

$\mathbf{N}$ ODE cooperation in multiterminal wireless networks has been shown to improve performance by providing increased robustness to channel variations and by enabling energy savings (see [1]-[7] and the references therein). A specific example of relay cooperation in multiterminal networks is the multi-access relay channel (MARC). The MARC is a

Manuscript received February 03, 2009; revised November 05, 2010; accepted November 18, 2010. Date of current version March 16, 2011. Part of this work was done while L. Sankar was with the WINLAB, Rutgers University, and Y. Liang was with Princeton University. L. Sankar (previously Sankaranarayanan) and H. V. Poor were supported in part by the National Science Foundation under Grant CNS-09-05398 and in part by the Air Force Office of Scientific Research under Grant FA9550-09-1-0643. L. Sankar was also supported in part by a Fellowship from the Princeton Council on Science and Technology. Y. Liang was supported in part by the National Science Foundation CAREER Award under Grant CCF-10-26565 and in part by the National Science Foundation under Grant CCF-10-26566. N. B. Mandayam was supported in part by the National Science Foundation under Grant CNS-0721826. The material in this paper was presented in part at the IEEE International Symposium on Information Theory, Nice, France, June 2007.

L. Sankar and H. V. Poor are with the Department of Electrical Engineering, Princeton University, Princeton, NJ 08544 USA.

Y. Liang is with the Department of Electrical Engineering and Computer Science, Syracuse University, Syracuse, NY 13244 USA.

N. B. Mandayam is with the WINLAB, Rutgers University, North Brunswick, NJ 08902 USA.

Communicated by M. C. Gastpar, Associate Editor for Shannon Theory.

Color versions of one or more of the figures in this paper are available online at http://ieeexplore.ieee.org.

Digital Object Identifier 10.1109/TIT.2011.2110690 network in which several users (source nodes) communicate with a single destination with the aid of a relay [8]. The coding strategies developed for the classical relay channel [9] extend readily to the MARC [10].

We consider a MARC with a half-duplex wireless relay that transmits and receives on two orthogonal channels. Specifically, we model a MARC with a half-duplex relay as an orthogonal MARC in which the relay receives on a channel over which all the sources transmit, and transmits to the destination on an orthogonal channel. ${ }^{1}$ This channel models a relay-inclusive uplink in a variety of networks such as wireless local area networks (LANs), cellular networks, and sensor networks. The study of wireless relay networks has focused on several performance aspects, including capacity (e.g. [1], [3], [9]), diversity (e.g., [2], [4], [12]), outage (e.g., [13]-[15]), and cooperative coding (e.g., [16], [17]). Equally pertinent is the problem of resource allocation in fading wireless channels in which both source and relay nodes can allocate their transmit powers to enhance a desired performance metric when the fading state information is available. Resource allocation for a variety of relay channels and networks has been studied in several papers, including [5], [13], and [18]-[20]. A common assumption in all these papers is that the source and relay nodes are subject to a total power constraint.

Resource allocation in multi-user relay networks has been studied recently in [21]-[23]. The authors in [21] and [23] consider a specific orthogonal model in which the sources time-duplex their transmissions and are aided in their transmissions by a half-duplex relay, while in [22] the optimal multi-user scheduling policy is determined under the assumption of a nonfading backhaul channel between the relay and destination. In contrast, in this paper, we consider a more general multi-access channel with a half-duplex (orthogonal) relay and model all internode wireless links as ergodic fading channels with perfect channel state information available at all nodes. Assuming a decode-and-forward (DF) relay, we develop the optimal source and relay power allocations and present conditions under which opportunistic time-duplexing of the users is optimal.

The orthogonal MARC is a multi-access generalization of the orthogonal relay channel studied in [6]; however, the optimal DF policies developed in [6] do not extend readily to maximize the DF sum-rate of the MARC. This is because unlike the single-user case, in order to determine the DF sum-rate for the

\footnotetext{
${ }^{1}$ Yet another class of orthogonal single-source half-duplex relay channels is defined in [11] in which the source and relay transmit in orthogonal bands. The source transmits in both bands, one of which is received at the relay and the other is received at the destination, such that the relay also transmits in the band received at the destination. In contrast to [11], we assume that all sources transmi in only one of the two orthogonal bands and the relay transmits in the other. Furthermore, we assume that signals in both bands are received at the destination.
} 
MARC, we need to consider the intersection of the two multi-access rate regions that result from decoding at both the relay and the destination. Here, we exploit the polymatroid properties of the two multi-access regions and use a single known lemma on the sum-rate of two intersecting polymatroids [24, chap. 46] to develop inner (DF) and outer bounds on the sum-rate and the rate region. We also specify the sub-class of orthogonal MARCs for which the DF bounds are tight.

A lemma in [24, chap. 46] enables us to classify polymatroid intersections broadly into two sets, namely, the sets of active and inactive cases. An active or an inactive case result when, in the region of intersection, the constraints on the $K$-user sum-rate at both receivers are active or inactive, respectively. In the sequel we show that inactive cases suggest partially clustered topologies in which a subset of users is clustered closer to one of the receivers while the complementary subset is closer to the remaining receiver. On the other hand, active cases can result from specific clustered topologies such as those in which all sources and the relay are clustered or those in which the relay and the destination are clustered, or more generally, from topologies that are either a combination of the two clustered models or of a clustered and a partially clustered model. For both the active and inactive cases, the polymatroid intersection lemma yields closed form expressions for the sum-rates which in turn allows one to develop the sum-rate optimal power allocations (policies).

We first develop the DF sum-rate maximizing power policies for a $K$-user orthogonal MARC. Using the polymatroid intersection lemma we show that the DF sum-rate averaged over all fading states is achieved by either one of five disjoint cases, two inactive and three active, or by a boundary case that lies at the boundary of an active and an inactive case. We develop the sum-rate for all cases and show that the sum-rate maximizing DF power policy either: 1) exploits the multi-user fading diversity to opportunistically schedule users analogously to the fading multiple access channel (MAC) [25], [26] though the optimal multi-user policies are not necessarily water-filling solutions, or 2) involves simultaneous water-filling over two independent point-to-point links.

Using similar techniques, we also develop the $K$-user DF rate region. Finally, we develop the cutset outer bounds on the sum-capacity. We show that DF achieves the sum-capacity for a class of orthogonal MARCs in which the sources and relay are clustered such that the outer bound on the $K$-user sum-rate at the destination dominates all other sum-rate outer bounds. We also show that DF achieves the capacity region when the cutset bounds at the destination are the dominant bounds for all rate points on the boundary of the outer bound rate region.

The paper is organized as follows. In Section II, we present the channel models and introduce polymatroids and a lemma on their intersections. In Section III we develop the DF rate region for ergodic fading orthogonal MARCs. In Section IV we develop the power policies that maximize the DF sum-rate for a two-user MARC. In Section IV we extend the analysis to the $K$-user orthogonal MARC as well as to nonorthogonal models. In Section VI, we present outer bounds and illustrate our results numerically. Finally, in Section VIII, we summarize our contributions.

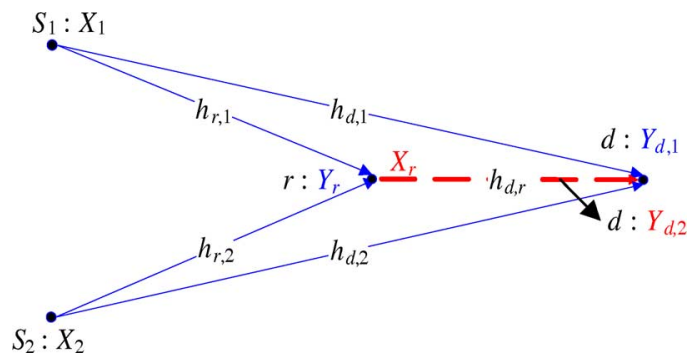

Fig. 1. A two-user orthogonal MARC.

\section{Channel Model And Preliminaries}

\section{A. Orthogonal Fading MARC}

A $K$-user MARC consists of $K$ source nodes numbered $1,2, \ldots, K$, a relay node $r$, and a destination node $d$. We write $\mathcal{K}=\{1,2, \ldots, K\}$ to denote the set of sources, $\mathcal{T}=\mathcal{K} \cup\{r\}$ to denote the set of transmitters, and $\mathcal{D}=\{r, d\}$ to denote the set of receivers. In an orthogonal MARC, the sources transmit to the relay and destination on one channel, say channel 1 , while the half-duplex relay transmits to the destination on an orthogonal channel 2 as shown in Fig. 1. Thus, a fraction $\theta$ of the total bandwidth resource is allocated to channel 1 while the remaining fraction $\bar{\theta}=1-\theta$ is allocated to channel 2 . In the fraction $\theta$, the source $k$, for all $k \in \mathcal{K}$, transmits the signal $X_{k}$ while the relay and the destination receive $Y_{r}$ and $Y_{d, 1}$ respectively. In the fraction $\bar{\theta}$, the relay transmits $X_{r}$ and the destination receives $Y_{d, 2}$ where the sources precede the relay in the transmission order. In each symbol time (channel use), we thus have

$$
\begin{aligned}
Y_{r} & =\sum_{k=1}^{K} H_{r, k} X_{k}+Z_{r} \\
Y_{d, 1} & =\sum_{k=1}^{K} H_{d, k} X_{k}+Z_{d, 1}, \quad \text { and } \\
Y_{d, 2} & =H_{d, r} X_{r}+Z_{d, 2}
\end{aligned}
$$

where $Z_{r}, Z_{d, 1}$, and $Z_{d, 2}$ are circularly symmetric complex Gaussian noise random variables with zero means and unit variances. We write $\underline{H}$ to denote a random vector of fading gains with entries $H_{m, k}$, for all $m \in \mathcal{D}$ and $k \in \mathcal{T}, k \neq m$. We use $\underline{h}$ to denote a realization of $\underline{H}$. We assume the fading process $\{\underline{H}\}$ is stationary and ergodic over time but not necessarily Gaussian. Note that the channel gains $H_{m, k}$ are not assumed to be independent, for all $m$ and $k$. We further assume that the parameter $\theta$ is fixed a priori, the same for every channel state, and is known at all nodes. As with the classical relay channel, the relay is assumed to be causal, and hence, the signal $X_{r}$ at the relay in each channel use depends causally only on the $Y_{r}$ received in the previous channel uses.

Over $n$ uses of the channel, the source and relay transmit sequences $\left\{X_{k, i}\right\}$ and $\left\{X_{r, i}\right\}$, respectively, which are constrained in power according to

$$
\sum_{i=1}^{n}\left|X_{k, i}\right|^{2} \leq n \bar{P}_{k}, \quad \text { for all } k \in \mathcal{T} .
$$


Since the sources and relay know the fading states of the links on which they transmit, they can allocate their transmitted signal powers according to the channel state information. A power policy $\underline{P}(\underline{h})$ is a mapping from the fading state space consisting of the set of all fading instantiations $\underline{h}$ to the set of positive real values in $\mathcal{R}_{+}^{K+1}$. The entries of $\underline{P}(\underline{h})$ are $P_{k}(\underline{h})$, the power policy at user $k$, for all $k \in \mathcal{T}$. While $\underline{P}(\underline{h})$ denotes the map for a particular fading instantiation, we write $\underline{P}(\underline{H})$ to explicitly describe the policy for the entire set of random channel states. Thus, we use the notation $\underline{P}(\underline{H})$ when averaging over all states or describing a collection of policies, one for every $\underline{h}$. The entries of $\underline{P}(\underline{H})$ are $P_{k}(\underline{H})$ for all $k \in \mathcal{T}$.

For an ergodic fading channel, (4) then simplifies to

$$
\mathbb{E}\left[P_{k}(\underline{H})\right] \leq \bar{P}_{k} \quad \text { for all } k \in \mathcal{T}
$$

where the expectation in (5) is over the distribution of $\underline{H}$. We denote the set of all feasible policies $\underline{P}(\underline{h})$, i.e., the power policies whose entries satisfy (5), by $\mathcal{P}$. Finally, we write $\underline{\bar{P}}$ to denote the vector of average power constraints with entries $\bar{P}_{k}$, for all $k \in \mathcal{T}$. Throughout the sequel, we also refer interchangeably to the transmit and receive fractions $\theta$ and $1-\theta$ as the first and second fractions, respectively.

We assume perfect channel state information (CSI) at the transmitters and receivers and a relatively long transmission time over which all fading states are seen. In practice channel estimation and feedback typically require a slowly varying channel as well as bandwidth and energy resources at the receivers. Despite such practical constraints, our assumption and the ensuing theoretical analysis defines the optimal performance bounds when the fading states are known perfectly at all nodes which in turn can serve as an upper bound on the performance of practical systems. Determining such performance bounds has led to fundamental results on ergodic capacities and optimal policies for many important ergodic channel models such as point-to-point [27], multiple access [25], [26], broadcast [28], and interference channels [29], [30].

Remark 1: We have chosen the bandwidth fraction $\theta$ to be fixed a priori to make the analysis and elucidation of our results easier; furthermore, such an assumption also models practical networks for which dynamic change of bandwidth fractions may not be straightforward or feasible. In general, however, $\theta$ can be chosen to maximize the sum-rate. Our analysis can be extended in a straightforward manner for the case of variable $\theta$, and where possible, we generalize our expressions to allow for this. Later in the sequel, we will illustrate our results for both fixed and varying $\theta$.

Remark 2: An alternate mechanism for half duplexed relay transmissions is to use independent time slots for the users and the relay. Such models have been considered for the MARC in [10] and, in general, for multiterminal relay networks in [21] and [23].

\section{B. Notation}

Before proceeding, we summarize the notation used in the sequel.
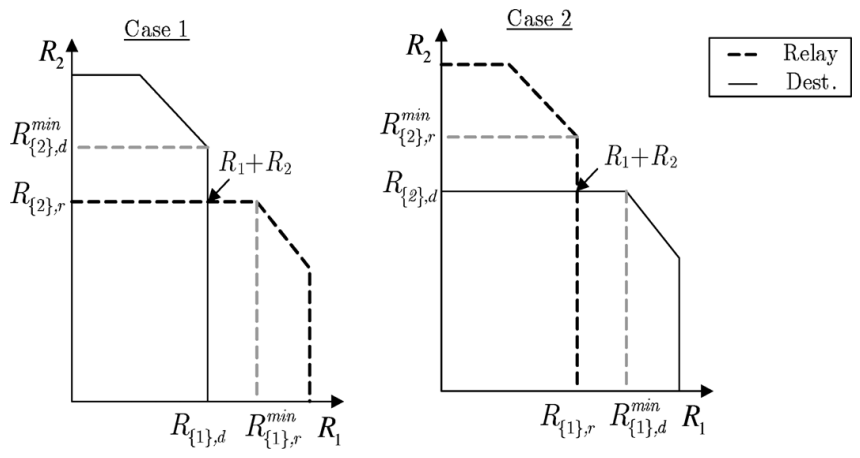

Fig. 2. Rate regions $R_{r}(\underline{P}(\underline{H}))$ and $R_{d}(\underline{P}(\underline{H}))$ and sum-rates for cases 1 and 2.

- Random variables (e.g., $H_{k, j}$ ) are denoted with uppercase letters and their realizations (e.g., $h_{k, j}$ ) with the corresponding lowercase letters.

- $\mathcal{C N}(0, \boldsymbol{\Sigma})$ denotes a circularly symmetric complex Gaussian distribution with zero mean and covariance $\boldsymbol{\Sigma}$.

- $\mathcal{K}=\{1,2, \ldots, K\}$ denotes the set of sources and $\mathcal{T}=$ $\mathcal{K} \cup\{r\}$ denotes the set of all transmitters.

- $\mathbb{E}(\cdot)$ denotes expectation; $C(x)$ denotes $\log (1+x)$ where the logarithm is to the base $2,(x)^{+}$denotes $\max (x, 0)$, $I(\cdot ; \cdot)$ denotes mutual information, $h(\cdot)$ denotes differential entropy, $\mathcal{X}_{\mathcal{S}}$ denotes $\left\{x_{k}: k \subset \mathcal{S}\right\}$, and $R_{\mathcal{S}}$ denotes $\sum_{k \in \mathcal{S}} R_{k}$ for any $\mathcal{S} \subseteq \mathcal{K}$.

- We use the usual notation for entropy and mutual information [31], [32] and take all logarithms to the base 2 so that our rate units are bits per channel use.

- Rate regions for a fixed $\theta$ are denoted with a superscript. $\underline{P}^{D F}(\underline{H})$ and $\underline{P}^{o b}(\underline{H})$ denote the sum-rate optimal power policies for DF and the cutset outer bounds, respectively.

\section{Polymatroids}

In the sequel, we use the properties of polymatroids to develop the ergodic sum-rate results. Polymatroids have been used to develop capacity characterizations for a variety of multipleaccess channel models including the MARC (see for e.g., [26], [33], [34]). We review the following definition of a polymatroid.

Definition 1: Let $\mathcal{K}=\{1,2, \ldots, K\}$ and $f=2^{K} \rightarrow \mathfrak{R}_{+}$be a set function. The polyhedron

$$
\begin{aligned}
\mathcal{B}(f) \equiv\left\{\left(R_{1}, R_{2}, \ldots, R_{K}\right): R_{\mathcal{S}} \leq f(\mathcal{S})\right. & \\
& \text { for all } \left.\mathcal{S} \subseteq \mathcal{K}, R_{k} \geq 0\right\}
\end{aligned}
$$

is a polymatroid if $f(\emptyset)=0$ (normalization), $f(\mathcal{S}) \leq f(\mathcal{P})$ if $\mathcal{S} \subset \mathcal{P}$ (monotonicity), and

$$
\begin{aligned}
f(\mathcal{S})+f(\mathcal{P}) & \\
& \geq f(\mathcal{S} \cup \mathcal{P})+f(\mathcal{S} \cap \mathcal{P}) \quad \text { (submodularity) }
\end{aligned}
$$

We use the following lemma on polymatroid intersections to develop optimal inner and outer bounds on the sum-rate for $K$-user orthogonal MARCs. 

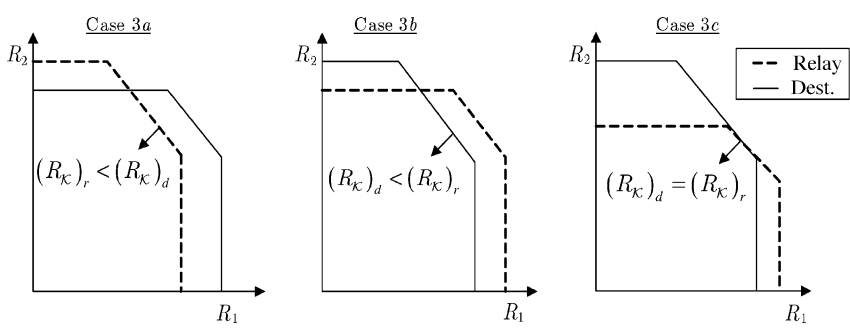

Fig. 3. Rate regions $R_{r}(\underline{P}(\underline{H}))$ and $R_{d}(\underline{P}(\underline{H}))$ and sum-rates for cases $3 a$, $3 b$, and $3 c$.

Lemma 1 ([24, p. 796, Cor. 46.1c]): Let $R_{\mathcal{S}} \leq f_{1}(\mathcal{S})$ and $R_{\mathcal{S}} \leq f_{2}(\mathcal{S})$, for all $\mathcal{S} \subseteq \mathcal{K}$, be two polymatroids. Then

$$
\max R_{\mathcal{K}}=\min _{\mathcal{S} \subseteq \mathcal{K}}\left(f_{1}(\mathcal{S})+f_{2}(\mathcal{K} \backslash \mathcal{S})\right) .
$$

Lemma 1 states that the maximum sum of $R_{k}$ over all $k$, denoted by $R_{\mathcal{K}}$, that results from the intersection of two polymatroids, $R_{\mathcal{S}} \leq f_{1}(\mathcal{S})$ and $R_{\mathcal{S}} \leq f_{2}(\mathcal{S})$, is given by the minimum of the two $K$-variable planes $f_{1}(\mathcal{K})$ and $f_{2}(\mathcal{K})$ only if both sums are at most as large as the sum of the orthogonal planes $f_{1}(\mathcal{S})$ and $f_{2}(\mathcal{K} \backslash \mathcal{S})$, for all $\emptyset \neq \mathcal{S} \subset \mathcal{K}$. We refer to the resulting intersection as belonging to the set of active cases (see Fig. 3 for an illustration of the active cases for $K=2$ ).

When there exists at least one $\emptyset \neq \mathcal{S} \subset \mathcal{K}$ for which the above condition is not true, an inactive case is said to result. For such cases, the maximum $K$-variable sum in (8) is the sum of two orthogonal rate planes achieved by two complementary subsets of users. As a result, the $K$-variable sum bounds $f_{1}(\mathcal{K})$ and $f_{2}(\mathcal{K})$ are no longer active for this case, and thus, the region of intersection is no longer a polymatroid with $2^{K}-1$ faces. For a $K$-user MARC, there are $2^{K}-2$ possible inactive cases. See Fig. 2 for an illustration of the inactive cases for $K=2$.

The intersection of two polymatroids can also result in a boundary case when for any $\mathcal{S} \subset \mathcal{K}, f_{1}(\mathcal{S})+f_{2}(\mathcal{K} \backslash \mathcal{S})$ is equal to one or both of the $K$-user sum-rate planes. The orthogonality of the planes $f_{1}(\mathcal{S})$ and $f_{2}(\mathcal{K} \backslash \mathcal{S})$ implies that no two inactive cases have a boundary, and thus, a boundary case arises only between an inactive and an active case. See Figs. 4 and 5 for an illustration of the boundary cases for $K=2$. Note that by definition, a boundary case is also an active case though for ease of exposition, throughout the sequel we explicitly distinguish between them. From (8), there are three possible active cases corresponding to the three cases in which the sum-rate plane at one of the receivers is smaller than, larger than, or equal to that at the other. In fact, the case in which the sum-rates are equal is also a boundary case between the other two active cases. Thus, there are a total of $\left(2^{K}-1\right)$ boundary cases for each active case.

In summary, the inactive set consists of all intersections for which the constraints on the two sum-rates are not active, i.e., no rate tuple on the sum-rate plane achieved at one of the receivers lies within or on the boundary of the rate region achieved at the other receiver. On the other hand, the intersections for which there exists at least one such rate tuple such that the two sum-rate constraints are active belong to the active set. Thus, by definition, the active set also includes those boundary cases between

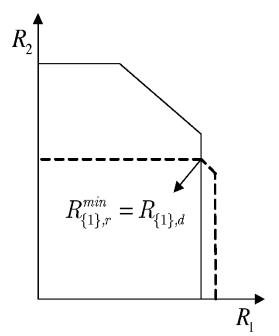

$\underline{\text { Case } 18 \text { Case } 3 a}$



$\underline{\text { Case } 18 \text { Case 3b }}$

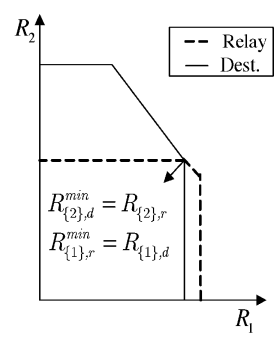

$\underline{\text { Case } 180 \text { Case } 3 c}$
Fig. 4. Rate regions $R_{r}(\underline{P}(\underline{H}))$ and $R_{d}(\underline{P}(\underline{H}))$ for cases $(1,3 \mathrm{a}),(1,3 \mathrm{~b})$, and $(1,3 \mathrm{c})$.

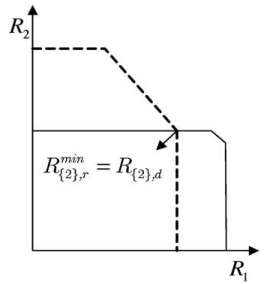

Case 28 Case $3 a$

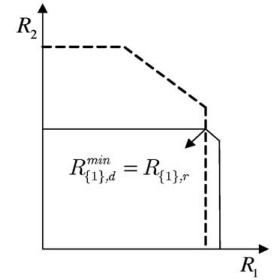

Case 283 Case $3 \mathrm{~b}$

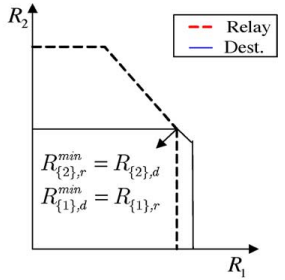

Case 28 Case $3 c$
Fig. 5. Rate regions $R_{r}(\underline{P}(\underline{H}))$ and $R_{d}(\underline{P}(\underline{H}))$ for cases (2,3a), (2,3b), and $(2,3 \mathrm{c})$.

the active and inactive cases for which there is exactly one such rate pair.

\section{ORTHOGONAL MARC: ERGODIC DF RATE REGION}

The DF rate regions for full-duplex discrete memoryless and Gaussian MARCs are developed in [3, Appendix A] (see [34] for a detailed proof) and [35], respectively. The DF rate bounds for the (half-duplex) orthogonal MARC can be obtained from those for the full-duplex MARC by incorporating this restriction via an additional conditioning on a mode random variable that models our orthogonal bandwidth constraint (see [36] for such modeling). In the interest of space, we refer the reader to [34] for the full-duplex bounds and present here directly the DF rate bounds for an orthogonal Gaussian MARC.

For the orthogonal Gaussian MARC with a fixed $\underline{h}$ and $\theta$ that are assumed to be known at all nodes, we consider Gaussian signaling at transmitter $k$ with zero mean and variance $P_{k}$ such that $X_{k} \sim C \mathcal{N}\left(0, P_{k}\right)$, for all $k \in \mathcal{T}$. Reliable decoding at the relay and at the destination in the appropriate fractions (the relay decodes using signals received in the fraction $\theta$ while the destination uses both fractions) requires that the transmitted rates satisfy the multiple access bounds at both receivers. The following proposition summarizes the resulting DF rate region.

Proposition 1: The DF rate region $\mathcal{R}_{D F}^{\theta}(\underline{P})$ for $K$-user orthogonal Gaussian MARCs with fixed channel states includes the set of all rate pairs $\left(R_{1}, R_{2}, \ldots, R_{K}\right)$ that satisfy

$$
\begin{aligned}
& R_{\mathcal{S}} \leq \min \left\{\theta C\left(\sum_{k \in \mathcal{S}}\left|h_{d, k}\right|^{2} P_{k} / \theta\right)\right. \\
&\left.+\bar{\theta} C\left(\left|h_{d, r}\right|^{2} P_{r} / \bar{\theta}\right), \quad \theta C\left(\sum_{k \in \mathcal{S}}\left|h_{r, k}\right|^{2} P_{k} / \theta\right)\right\} \\
& \text { for all } \mathcal{S} \subseteq \mathcal{K} .
\end{aligned}
$$


For a stationary and ergodic process $\{\underline{H}\}$, the channel in (1)-(3) can be modeled as a set of parallel Gaussian orthogonal MARCs, one for each fading instantiation $\underline{h}$. For a power policy $\underline{P}(\underline{H})$, assuming Gaussian signaling at the transmitters, the DF rate bounds for this ergodic fading channel are given as a weighted average of the rate bounds achieved in each fading state (the parallel orthogonal Gaussian MARC) where the weights denote the probabilities of occurence of the fading states. Considering the rate regions over all $\underline{P}(\underline{H}) \in \mathcal{P}$ yields the ergodic fading DF rate region, $\mathcal{R}_{D F}^{\theta}(\underline{\bar{P}})$, where $\underline{\bar{P}}$ is defined in Section II as a vector of average power constraints at all transmitters (sources and relay). The ergodic fading DF rate region, $\mathcal{R}_{D F}^{\theta}(\underline{\bar{P}})$, for a fixed bandwidth fraction $\theta$, is summarized by the following theorem.

Theorem 1: The DF rate region $\mathcal{R}_{D F}^{\theta}(\underline{\bar{P}})$ of a $K$-user ergodic fading orthogonal Gaussian MARC is

$$
\mathcal{R}_{D F}^{\theta}(\underline{\bar{P}})=\bigcup_{\underline{P}(\underline{H}) \in \mathcal{P}}\left\{\mathcal{R}_{r}^{\theta}(\underline{P}(\underline{H})) \cap \mathcal{R}_{d}^{\theta}(\underline{P}(\underline{H}))\right\}
$$

where

$$
\begin{aligned}
& \mathcal{R}_{r}(\underline{P}(\underline{H})) \\
&=\left\{\left(R_{1}, R_{2}, \ldots, R_{K}\right):\right. \\
&\left.R_{\mathcal{S}} \leq \mathbb{E}\left[\theta C\left(\sum_{k \in \mathcal{S}}\left|H_{r, k}\right|^{2} P_{k}(\underline{H}) / \theta\right)\right]\right\} \\
& \text { for all } \mathcal{S} \subseteq \mathcal{K}
\end{aligned}
$$

and

$$
\begin{aligned}
\mathcal{R}_{d}(\underline{P}(\underline{H})) \\
=\left\{\left(R_{1}, R_{2}, \ldots, R_{K}\right):\right. \\
\quad R_{\mathcal{S}} \leq \mathbb{E}\left[\theta C\left(\sum_{k \in \mathcal{S}}\left|H_{d, k}\right|^{2} P_{k}(\underline{H}) / \theta\right)\right. \\
\left.\left.\quad+\bar{\theta} C\left(\left|H_{d, r}\right|^{2} P_{r}(\underline{H}) / \bar{\theta}\right)\right], \quad \text { for all } \mathcal{S} \subseteq \mathcal{K}\right\} .
\end{aligned}
$$

Proof: The proof follows from the observation that the channel in (1)-(3) can be modeled as a set of parallel Gaussian orthogonal MARCs, one for each fading instantiation $\underline{h}$ and the fact that independent signals are transmitted in each parallel channel. We use the argument $\underline{P}(\underline{H})$ in denoting the rate regions since the rates are averaged over the channel states. The DF rate region, $\mathcal{R}_{D F}^{\theta}$, is given by the union of such intersections, one for each $\underline{P}(\underline{H}) \in \mathcal{P}$. The convexity of $\mathcal{R}_{D F}^{\theta}$ follows from the convexity of the set $\mathcal{P}$ and the concavity of the $\log$ function.

Proposition 2: $\mathcal{R}_{r}^{\theta}(\underline{P}(\underline{H}))$ and $\mathcal{R}_{d}^{\theta}(\underline{P}(\underline{H}))$ are polymatroids.

Proof: In [34, Sec. IV.B], it is shown that for each choice of the input distribution, the DF rate region is an intersection of two polymatroids, one resulting from the bounds at the relay and the other from the bounds at the destination. For the orthogonal Gaussian MARC, the bounds in (11) and (12) involve a weighted sum of mutual information expressions; using the same approach as in [34, Sec. IV.B], the submodularity of these expressions can be verified in a straightforward manner.
Remark 3: The DF rate region is obtained using block Markov encoding at the sources. For the ergodic fading model, the rates in Theorem 1 are obtained assuming that each block is large enough to contain all fading instantiations in an ergodic manner.

Remark 4: For the case where $\theta$ can be varied, the DF rate region $\mathcal{R}_{D F}$ is obtained as a union of $\mathcal{R}_{D F}^{\theta}$ over all feasible values of $\theta$, i.e., $\mathcal{R}_{D F}=\bigcup_{\theta \in(0,1)} \mathcal{R}_{D F}^{\theta}$.

In the following sections, we first develop the sum-rate optimal DF power policies for the two-user case and then generalize it for the $K$-user case.

\section{TWO-USER ORTHOGONAL MARC: DF SUM-RATE OPTIMAL POWER POLICY}

For ease of notation, throughout the sequel, we write $R_{\mathcal{A}, j}$ to denote the sum-rate bound on the users in $\mathcal{A}$ and $R_{\mathcal{A}, j}^{\min }$ to denote the sum-rate obtained by successively decoding the users in $\mathcal{A}$ before decoding those in $\mathcal{K} \backslash \mathcal{A}$ at receiver $j=r, d$, i.e., $R_{\mathcal{A}, j}^{\min }=R_{\mathcal{K}, j}-R_{\mathcal{K} \backslash \mathcal{A}, j}$. See Fig. 2 for an illustration. For the two-user case, $R_{\mathcal{K}, j}$ and $R_{\mathcal{A}, j}$, for all $\mathcal{A} \subset \mathcal{K}$ are given by the sum-rate and single-user bounds in (11) and (12) at the relay and destination, respectively.

The region $\mathcal{R}_{D F}^{\theta}$ in (10) is a union of the intersections of the regions $\mathcal{R}_{r}^{\theta}(\underline{P}(\underline{H}))$ and $\mathcal{R}_{d}^{\theta}(\underline{P}(\underline{H}))$ achieved at the relay and destination respectively, where the union is over all $\underline{P}(\underline{H}) \in \mathcal{P}$. Since $\mathcal{R}_{D F}^{\theta}$ is convex, each point on the boundary of $\mathcal{R}_{D F}^{\theta}$ is obtained by maximizing the weighted sum $\mu_{1} R_{1}+\mu_{2} R_{2}$ over all $\underline{P}(\underline{H}) \in \mathcal{P}$, and for all $\mu_{1}>0, \mu_{2}>0$. Specifically, we determine the optimal policy $\underline{P}^{*}(\underline{H})$ that maximizes the sumrate $R_{1}+R_{2}$ when $\mu_{1}=\mu_{2}$. Observe from (10) that every point on the boundary of $\mathcal{R}_{D F}^{\theta}$ results from the intersection of the polymatroids (pentagons) $\mathcal{R}_{r}^{\theta}(\underline{P}(\underline{H}))$ and $\mathcal{R}_{d}^{\theta}(\underline{P}(\underline{H}))$ for some $\underline{P}(\underline{H})$. In Figs. 2 and 3 we illustrate the five possible choices for the sum-rate resulting from such an intersection for a two-user MARC of which two belong to the inactive set and three to the active set.

The inactive set consists of cases 1 and 2 in which user 1 achieves a significantly larger rate at the relay and destination, respectively, than it does at the other receiver; and vice-versa for user 2 . The active set includes cases $3 a, 3 b$, and $3 c$ shown in Fig. 2 in which the sum-rate at relay $r$ is smaller, larger, or equal, respectively, to that achieved at the destination $d$. The three boundary cases between case 1 and the three active cases are shown in Fig. 4 while the remaining three between case 2 and the active cases are shown in Fig. 5. We denote a boundary case as case $(l, n), l=1,2, n=3 a, 3 b, 3 c$.

We write $\mathcal{B}_{i} \subseteq \mathcal{P}$ and $\mathcal{B}_{l, n} \subseteq \mathcal{P}$ to denote the set of power policies that achieve case $i, i=1,2,3 a, 3 b, 3 c$, and case $(l, n)$, $l=1,2, n=3 a, 3 b, 3 c$, respectively. We show in the sequel that the optimization is simplified when the conditions for each case are defined such that the sets $\mathcal{B}_{i}$ and $\mathcal{B}_{l, n}$ are disjoint for all $i, l$, and $n$, and thus, are either open or half-open sets such that no two sets share a boundary. Observe that cases 1 and 2 do not share a boundary since such a transition (see Fig. 2) requires passing through case $3 a$ or $3 b$ or $3 c$. Finally, note that Fig. 3 illustrates two specific $\mathcal{R}_{r}^{\theta}$ and $\mathcal{R}_{d}^{\theta}$ regions for $3 a, 3 b$, and $3 c$. For ease of exposition, we write $\mathcal{B}_{3}=\mathcal{B}_{3 a} \cup \mathcal{B}_{3 b} \cup \mathcal{B}_{3 c}$. 
In general, the occurrence of any one of the disjoint cases depends on both the channel statistics and the policy $\underline{P}(\underline{H})$. Since it is not straightforward to know a priori the power allocations that achieve a certain case, we maximize the sum-rate for each case over all allocations in $\mathcal{P}$ and explicitly check whether the optimizing power allocation indeed results in the corresponding case. In the following, we will argue that this can be true for only one case, and the optimizing power policy for this case is the unique solution that achieves the optimal sum-rate.

We write $\underline{P}^{(i)}(\underline{H})$ and $\underline{P}^{(l, n)}(\underline{H})$ to denote the optimal solution for case $i$ and case $(l, n)$, respectively. Explicitly including boundary cases ensures that the sets $\mathcal{B}_{i}$ and $\mathcal{B}_{l, n}$ are disjoint for all $i$ and $(l, n)$, i.e., these sets are either open or half-open sets such that no two sets share a power policy in common. This in turn simplifies the convex optimization as follows.

Consider case $i$. The optimal $\underline{P}^{(i)}(\underline{H})$ is first determined by maximizing the sum rate for this case over all $\mathcal{P}$. The resulting sum-rate optimal $\underline{P}^{(i)}(\underline{H})$ must satisfy the conditions for case $i$, i.e., we require $\underline{P}^{(i)}(\underline{H}) \in \mathcal{B}_{i}$. If $\underline{P}^{(i)}(\underline{H}) \in \mathcal{B}_{i}$, the optimality of $\underline{P}^{(i)}(\underline{H})$ follows from the fact that the rate function for each case is strictly concave and that the sets $\mathcal{B}_{i}$ and $\mathcal{B}_{l, n}$ are disjoint for all $i$ and $(l, n)$ as a result of which $\underline{P}^{(i)}(\underline{H})$ does not maximize the sum-rate for any other case. On the other hand, when $\underline{P}^{(i)}(\underline{H}) \notin \mathcal{B}_{i}$, we now argue that $R_{1}+R_{2}$ achieves its maximum outside $\mathcal{B}_{i}$. The proof again follows from the fact that $R_{1}+R_{2}$ for all cases is a strictly concave function of $\underline{P}(\underline{H})$ for all $\underline{P}(\underline{H}) \in \mathcal{P}$. Thus, when $\underline{P}^{(i)}(\underline{H}) \notin \mathcal{B}_{i}$, for every $\underline{P}(\underline{H}) \in \mathcal{B}_{i}$ there exists a $\underline{P}^{\prime}(\underline{H}) \in \mathcal{B}_{i}$ with a larger sum-rate. Combining this with the fact that the sum-rate expressions are continuous while transitioning from one case to another at the boundary of the open set $\mathcal{B}_{i}$, ensures that the maximal sum-rate is achieved by some $\underline{P}(\underline{H}) \notin \mathcal{B}_{i}$. Similar arguments justify maximizing the optimal policy for each case over all $\mathcal{P}$. Due to the strict concavity of the logarithm function, a unique $\underline{P}^{(i)}(\underline{H})$ or $\underline{P}^{(l, n)}(\underline{H})$ will satisfy the conditions for its case. The optimal $\underline{P}^{*}(\underline{H})$ is given by this $\underline{P}^{(i)}(\underline{H})$ or $\underline{P}^{(l, n)}(\underline{H})$.

The optimization problem for case $i$ or case $(l, n)$ is given by

$S_{D F}^{\theta}=\max _{\underline{P}(\underline{H}) \in \mathcal{P}} S_{\theta}^{(i)}$ or $\max _{\underline{P}(\underline{H}) \in \mathcal{P}} S_{\theta}^{(l, n)}$
s.t. $\quad \mathbb{E}\left[P_{k}(\underline{H})\right] \leq \bar{P}_{k}, \quad k=1,2, r$
$P_{k}(\underline{h}) \geq 0, \quad k=1,2, r, \quad$ for all $\underline{h}$

where

$$
\begin{aligned}
& S_{\theta}^{(1)}=R_{\{1\}, d}+R_{\{2\}, r} \\
& S_{\theta}^{(2)}=R_{\{1\}, r}+R_{\{2\}, d} \\
& S_{\theta}^{(3 a)}=R_{\mathcal{K}, r} \\
& S_{\theta}^{(3 b)}=R_{\mathcal{K}, d} \\
& S_{\theta}^{(3 c)}=(1-\alpha) R_{\mathcal{K}, r}+\alpha R_{\mathcal{K}, d}, \alpha \text { s.t. } R_{\mathcal{K}, r}=R_{\mathcal{K}, d} \\
& S_{\theta}^{(l, n)}=(1-\beta) S_{\theta}^{(l)}+\beta S_{\theta}^{(n)}, \beta \text { s.t. } S_{\theta}^{(l)}=S_{\theta}^{(n)}
\end{aligned}
$$

and the subscript $\theta$ in $S^{(\cdot)}$ indicates that $\theta$ is fixed.

Let $S_{\mathrm{MAC}}$ denote the sum-capacity that the two users achieve at the destination in the absence of the relay, i.e., $\theta=1$ (or $\theta=0$ and $\left.\bar{P}_{r}=0\right)$. From [25] and [26], we know that the optimizing policy $P_{\mathrm{MAC}}(\underline{H})$ simplifies to multi-user opportunistic waterfilling. For a fixed $\theta$ the maximum achievable sum-rate is then given by

$$
\max \left(S_{\mathrm{MAC}}, S_{D F}^{\theta}\right) .
$$

More generally, when all feasible values of the bandwidth fraction $\theta$ are allowed, the maximum achievable sum-rate is given by

$$
\max \left(S_{\mathrm{MAC}}, \max _{\theta \in(0,1)} S_{D F}^{\theta}\right) .
$$

Remark 5: In (16), allowing the range of $\theta$ to include $\theta=1$ covers the MAC without relay case.

Throughout the discussion below, we assume that $\theta$ is fixed, and therefore, (15) is used to determine the maximal sum-rate. For the case in which $S_{\mathrm{MAC}}$ is larger, it suffices to not allocate any bandwidth resources for relay transmission and simply communicate directly with the destination, i.e., $\theta=1$. While this may hold for any case, it is particularly possible for cases $3 a,(1,3 a)$, and $(2,3 a)$, where the multiple access link to the relay is the bottleneck link. We now determine the sum-rate maximizing policy for each case and assume that (15) is always used to determine the maximal sum-rate.

For each case, we determine the optimal policy using Lagrange multipliers and the Karush-Kuhn-Tucker (KKT) conditions [37, 5.5.3]. A detailed analysis is developed in the Appendix and we summarize the KKT conditions and the optimal policies for all cases below. From (14), the KKT conditions for each case $x, x=i,(l, n)$, for all $i$ and $(l, n)$ are given as

$$
\begin{array}{r}
f_{k}^{(x)}(\underline{P}(\underline{h}))-\nu_{k} \ln 2 \leq 0, \text { with equality for } P_{k}(\underline{h})>0 \\
k=1,2, r, \quad \text { for all } \underline{h}
\end{array}
$$

where $\nu_{k}$, for all $k=1,2, r$, are dual variables chosen to satisfy the power constraints in (13) and $f_{k}^{(x)}(\cdot)$ will be defined later for each case. Specializing the KKT conditions for each case, we obtain the optimal policies for each case as summarized below following which we list the conditions that the optimal policy for each case needs to satisfy.

Case 1: The functions $f_{k}^{(1)}(\underline{P}(\underline{h})), k=1,2, r$, in (17) for case 1 are

$$
\begin{aligned}
f_{k}^{(1)}(\underline{P}(\underline{h})) & =\frac{\left|h_{m, k}\right|^{2}}{\left(1+\left|h_{m, k}\right|^{2} P_{k}(\underline{h}) / \theta\right)} \\
f_{r}^{(1)}(\underline{P}(\underline{h})) & =\frac{\mid k, m)=(1, d),(2, r)}{\left(1+\left|h_{d, r}\right|^{2} P_{k}(\underline{h}) / \bar{\theta}\right)} .
\end{aligned}
$$

It is straightforward to verify that these KKT conditions simplify to

$$
\begin{aligned}
P_{k}^{(1)}(\underline{h})= & \left(\frac{\theta}{\nu_{k} \ln 2}-\frac{\theta}{\left|h_{m, k}\right|^{2}}\right)^{+} \\
& (k, m)=(1, d),(2, r)
\end{aligned}
$$

and

$$
P_{r}^{(1)}(\underline{h})=\left(\frac{\bar{\theta}}{\nu_{r} \ln 2}-\frac{\bar{\theta}}{\left|h_{d, r}\right|^{2}}\right)^{+}
$$

Case 2: From (14), since $S^{(2)}$ can be obtained from $S^{(1)}$ by interchanging the user indices 1 and 2 , the functions 
$f_{k}^{(2)}(\underline{P}(\underline{h}))$, and hence, the KKT conditions for this case can be obtained by replacing the superscript (1) by (2) and using the pairs $(k, m)=(1, r),(2, d)$ in (18)-(20). The resulting optimal policies are

$$
\begin{aligned}
& P_{r}^{(2)}(\underline{h})=P_{r}^{(1)}(\underline{h}), \quad \text { for all } \underline{h}, \text { and } \\
& P_{k}^{(2)}(\underline{h})=\left(\frac{\theta}{\nu_{k} \ln 2}-\frac{\theta}{\left|h_{m, k}\right|^{2}}\right)^{+} \\
& \quad(k, m)=(1, r),(2, d) .
\end{aligned}
$$

Case 3a: The functions $f_{k}^{(3 a)}(\underline{P}(\underline{h})), k=1,2$, satisfying the KKT conditions in (17) are

$$
\begin{array}{r}
f_{k}^{(3 a)}(\underline{P}(\underline{h}))=\left|h_{r, k}\right|^{2} /\left(1+\sum_{k=1}^{2}\left|h_{r, k}\right|^{2} P_{k}(\underline{h}) / \theta\right) \\
k=1,2 .
\end{array}
$$

Since this case maximizes the multi-access sum-rate at the relay, the optimal user policies are multi-user opportunistic waterfilling solutions given by

$$
\begin{aligned}
\frac{\left|h_{r, 1}\right|^{2}}{v_{1}}>\frac{\left|h_{r, 2}\right|^{2}}{\nu_{2}}: P_{2}^{(3 a)} & =0, P_{1}^{(3 a)}(\underline{h}) \\
& =\left(\frac{\theta}{\nu_{1} \ln 2}-\frac{\theta}{\left|h_{r, 1}\right|^{2}}\right)^{+} \\
\frac{\left|h_{r, 1}\right|^{2}}{v_{1}}<\frac{\left|h_{r, 2}\right|^{2}}{\nu_{2}}: P_{1}^{(3 a)}(\underline{h}) & =0, P_{2}^{(3 a)} \\
& =\left(\frac{\theta}{\nu_{2} \ln 2}-\frac{\theta}{\left|h_{r, 2}\right|^{2}}\right)^{+} \\
\frac{\left|h_{r, 1}\right|^{2}}{v_{1}} & =\frac{\left|h_{r, 2}\right|^{2}}{\nu_{2}}: P_{1}^{(3 a)}(\underline{h}) \\
& =P_{2}^{(3 a)}(\underline{h}) \\
& =\left(\frac{\theta / 2}{\nu_{2} \ln 2}-\frac{\theta / 2}{\left|h_{r, 2}\right|^{2}}\right)^{+}
\end{aligned}
$$

Thus, from (24), we see that the sum-rate $S^{(3 a)}$ is maximized when each user exploits knowledge of the channel states to $o p$ portunistically schedule its transmissions when its fading state is better than that of the other. Finally, while the relay power does not explicitly appear in the optimization, since this case results when the sum-rate at the relay is smaller than that at the destination, choosing the optimal relay policy to maximize the sum-rate at the destination, i.e., $P_{r}^{(3 a)}(\underline{h})=P_{r}^{(1)}(\underline{h})$, will ensure the case conditions. However, it is worth noting that forwarding via the relay is desirable for this case only if

$$
S_{\theta}^{(3 a)}\left(\underline{P}^{(3 a)}(\underline{H})\right)>S_{\mathrm{MAC}}\left(\underline{P}^{(w f)}(\underline{H})\right)
$$

is satisfied for the chosen $\theta$ (or some $\theta$ when $\theta$ is allowed to vary). Otherwise, it is better to transmit directly to the destination by setting $\theta=1$, i.e., not use the relay.

Case $3 b$ : The functions $f_{k}^{(3 b)}(\underline{P}(\underline{h})), k=1,2$, satisfying the KKT conditions in (17) can be obtained from (23) by replacing the subscript ' $r$ ' by ' $d$ ' in (23) while $f_{r}^{(3 b)}(\underline{P}(\underline{h}))=$ $f_{r}^{(1)}(\underline{P}(\underline{h}))$. Thus, this case maximizes the multi-access sum- rate at the destination and the optimal user policies are multiuser opportunistic water-filling solutions given by

$$
\begin{aligned}
\frac{\left|h_{d, 1}\right|^{2}}{v_{1}}>\frac{\left|h_{d, 2}\right|^{2}}{\nu_{2}}: P_{2}^{(3 b)} & =0, P_{1}^{(3 b)}(\underline{h}) \\
& =\left(\frac{\theta}{\nu_{1} \ln 2}-\frac{\theta}{\left|h_{d, 1}\right|^{2}}\right)^{+} \\
\frac{\left|h_{d, 1}\right|^{2}}{v_{1}}<\frac{\left|h_{d, 2}\right|^{2}}{\nu_{2}}: P_{1}^{(3 b)}(\underline{h}) & =0, P_{2}^{(3 b)} \\
& =\left(\frac{\theta}{\nu_{2} \ln 2}-\frac{\theta}{\left|h_{d, 2}\right|^{2}}\right)^{+} \\
\frac{\left|h_{d, 1}\right|^{2}}{v_{1}}=\frac{\left|h_{d, 2}\right|^{2}}{\nu_{2}}: P_{1}^{(3 b)}(\underline{h}) & =P_{2}^{(3 b)}(\underline{h}) \\
& =\left(\frac{\theta / 2}{\nu_{2} \ln 2}-\frac{\theta / 2}{\left|h_{d, 2}\right|^{2}}\right)^{+}
\end{aligned}
$$

while the optimal relay policy is a water-filling solution $P_{r}^{(3 b)}(\underline{h})=P_{r}^{(1)}(\underline{h})$.

Case $3 c$ : The functions $f_{k}^{(3 c)}(\underline{P}(\underline{h})), k=1,2, r$, satisfying the KKT conditions in (17) are given as

$$
\begin{aligned}
& f_{k}^{(3 c)}(\underline{P}(\underline{h}))=(1-\alpha) f_{k}^{(3 a)}(\underline{P}(\underline{h}))+\alpha f_{k}^{(3 b)}(\underline{P}(\underline{h})) \\
& f_{r}^{(3 c)}(\underline{P}(\underline{h}))=\alpha f_{r}^{(3 b)}(\underline{P}(\underline{h})), \quad k=r
\end{aligned}
$$

where the Lagrange multiplier $\alpha$ accounts for the boundary condition

$$
R_{\mathcal{K}, d}(\underline{P}(\underline{H}))=R_{\mathcal{K}, r}(\underline{P}(\underline{H}))
$$

and the optimal policy $\underline{P}^{(3 c)}(\underline{H}) \in \mathcal{B}_{3 c}$ satisfies (29) where $\mathcal{B}_{3 c}$ is the set of $\underline{P}(\underline{H})$ that satisfy (29). In the Appendix, using the KKT conditions we show that the optimal user policies are opportunistic in form and are given by

$$
\begin{aligned}
& f_{1}^{(3 c)} / \nu_{1}>f_{2}^{(3 c)} / \nu_{2}: \\
& P_{1}^{(3 c)}(\underline{h})=\left(\text { root of }\left.F_{1}^{(3 c)}\right|_{P_{2}=0}\right)^{+} \\
& P_{2}^{(3 c)}(\underline{h})=0 \\
& f_{1}^{(3 c)} / \nu_{1}<f_{2}^{(3 c)} / \nu_{2}: \\
& P_{1}^{(3 c)}(\underline{h})=0 \\
& P_{2}^{(3 c)}(\underline{h})=\left(\text { root of }\left.F_{2}^{(3 c)}\right|_{P_{1}=0}\right)^{+} \\
& f_{1}^{(3 c)} / \nu_{1}=f_{2}^{(3 c)} / \nu_{2}: \\
& P_{1}^{(3 c)}(\underline{h}) \text { and } P_{2}^{(3 c)}(\underline{h}) \text { obtained }
\end{aligned}
$$$$
\text { using iterative non-water-filling }
$$

where we write

$$
F_{k}^{(3 c)}=f_{k}^{(3 c)}-\nu_{k} \ln 2 \quad k=1,2 .
$$

Analogous to cases $3 a$ and $3 b$, the scheduling conditions in (30) depend on both the channel states and the water-filling levels $\nu_{k}$ at both users. However, the conditions in (30) also depend on the power policies, and thus, the optimal solutions are no longer water-filling solutions. In the Appendix we show that the optimal user policies can be computed using an iterative nonwater-filling algorithm which starts by fixing the power policy 
of one user, computing that of the other, and vice-versa until the policies converge to the optimal policy. The iterative algorithm is computed for increasing values of $\alpha \in(0,1)$ until the optimal policy satisfies (29) at the optimal $\alpha^{*}$. The proof of convergence is detailed in the Appendix. Finally, since $f_{r}^{(3 c)}=\alpha f_{r}^{(1)}$, the relay's optimal policy simplifies to the water-filling solution given by

$$
P_{r}^{(3 c)}(\underline{h})=\left(\frac{\alpha^{*} \bar{\theta}}{\nu_{r} \ln 2}-\frac{\bar{\theta}}{\left|h_{d, r}\right|^{2}}\right)^{+} .
$$

Boundary Cases $(l, n)$ : A boundary case $(l, n)$ results when

$$
S^{(l)}=S^{(n)} \quad l=1,2, \text { and } n=3 a, 3 b, 3 c .
$$

Recall that $S^{(l)}$ and $S^{(n)}$ are sum-rates for an inactive case $l$, and an active case $n$, respectively. Thus, in addition to the constraints in (13), the maximization problem for these cases includes the additional constraint in (33). For all except the two cases where $n=3 c$, the equality condition in (29) is represented by a Lagrange multiplier $\alpha$. The two cases with $n=3 c$ have two Lagrange multipliers $\alpha_{1}$ and $\alpha_{2}$ to also account for both the equality condition in (29) and the condition $S^{(3 a)}=S^{(3 b)}$.

For the different boundary cases, the functions $f_{k}^{(l, n)}(\underline{P}(\underline{h}))$, $k=1,2$, satisfying the KKT conditions in (17) are given as

$$
\begin{aligned}
f_{k}^{(l, n)}(\underline{P}(\underline{h}))= & (1-\alpha) f_{k}^{(l)}(\underline{P}(\underline{h}))+\alpha f_{k}^{(n)}(\underline{P}(\underline{h})) \\
k=1,2, n \neq 3 c & \\
f_{k}^{(l, 3 c)}(\underline{P}(\underline{h}))= & \left(1-\alpha_{1}-\alpha_{2}\right) f_{k}^{(l)}(\underline{P}(\underline{h})) \\
& +\alpha_{2} f_{k}^{(3 a)}(\underline{P}(\underline{h}))+\alpha_{1} f_{k}^{(3 b)}(\underline{P}(\underline{h})) \\
k=1,2 & (35) \\
f_{r}^{(l, n)}(\underline{P}(\underline{h}))= & (1-\alpha) f_{r}^{(l)}(\underline{P}(\underline{h})), \quad n=3 a \\
f_{r}^{(l, n)}(\underline{P}(\underline{h}))= & (1-\alpha) f_{r}^{(l)}(\underline{P}(\underline{h}))+\alpha f_{r}^{(n)}(\underline{P}(\underline{h})) \\
f_{r}^{(l, n)}(\underline{P}(\underline{h}))= & \left(1-\alpha_{1}-\alpha_{2}\right) f_{r}^{(l)}(\underline{P}(\underline{h}))+\alpha_{1} f_{r}^{(3 b)}(\underline{P}(\underline{h})) \\
n=3 c . \quad & (38)
\end{aligned}
$$

For ease of exposition and brevity, we summarize the KKT conditions and the optimal policies for case $(1,3 a)$. In the Appendix, using the KKT conditions we show that the optimal user policies $P_{k}^{(1,3 a)}(\underline{h})$ are opportunistic in form and are given by

$$
\begin{aligned}
& \frac{f_{1}^{(1,3 a)}}{\nu_{1}}>\frac{f_{2}^{(1,3 a)}}{\nu_{2}}: \\
& P_{1}^{(1,3 a)}(\underline{h})=\left(\text { root of }\left.F_{1}^{(1,3 a)}\right|_{P_{2}=0}\right)^{+} \\
& P_{2}(\underline{h})=0 \\
& \frac{f_{1}^{(1,3 a)}}{\nu_{1}}<\frac{f_{2}^{(1,3 a)}}{\nu_{2}}: \\
& P_{1}^{(1,3 a)}(\underline{h})=0 \\
& P_{2}(\underline{h})=\left(\text { root of }\left.F_{2}^{(1,3 a)}\right|_{P_{1}=0}\right)^{+} \\
& \frac{f_{1}^{(1,3 a)}}{\nu_{1}}=\frac{f_{2}^{(1,3 a)}}{\nu_{2}}: \\
& P_{1}^{(1,3 a)}(\underline{h}) \text { and } P_{2}(\underline{h}) \text { solved jointly } \\
& \text { using iterative non-water-filling }
\end{aligned}
$$

where $F_{k}^{(1,3 a)}=f_{k}^{(1,3 a)}-\nu_{k} \ln 2$, for $k=1,2$. As in case $3 c$, the optimal policies take an opportunistic nonwater-filling form and in fact can be obtained by an iterative nonwater-filling algorithm as described for case $3 c$. Furthermore, analogously to case $3 c$, the user policies are computed for increasing values of $\alpha \in(0,1)$ until the optimal policy satisfies (33) at the optimal $\alpha^{*}$. The optimal $P_{r}^{(1,3 a)}(\underline{h})=\alpha^{*} P_{r}^{(1)}(\underline{h})$ is a water-filling solution.

The optimal policies for all other boundary cases can be obtained similarly as detailed in the Appendix and can be computed using the iterative algorithm detailed in the Appendix. Specifically, for cases $(l, 3 c), l=1,2$, the iterative algorithm is computed for increasing values of $\alpha_{1}, \alpha_{2} \in(0,1)$ until the optimal policy satisfies (33) and (29) at the optimal $\alpha_{1}^{*}$ and $\alpha_{2}^{*}$, respectively. For all boundary cases, the optimal user policies are opportunistic nonwater-filling solutions while those for the relay are water-filling solutions. Finally, the sum-rate maximizing policy for any case is the optimal policy only if it satisfies the conditions for that case. The conditions for the cases are

$$
\begin{aligned}
& \underline{\text { Case } 1}: R_{\{1\}, d}<R_{\{1\}, r}^{\min } \text { and } R_{\{2\}, r}<R_{\{2\}, d}^{\min } \\
& \text { Case } 2: R_{\{1\}, r}<R_{\{1\}, d}^{\min } \text { and } R_{\{2\}, d}<R_{\{2\}, r}^{\min } \\
& \underline{\text { Case } 3 a}: R_{\mathcal{K}, r}<R_{\mathcal{K}, d}, R_{\{1\}, r}^{\min }<R_{\{1\}, d} \\
& \text { and } R_{\{2\}, r}^{\min }<R_{\{2\}, d} \\
& \underline{\text { Case } 3 b}: R_{\mathcal{K}, r}>R_{\mathcal{K}, d}, R_{\{2\}, d}^{\min }<R_{\{2\}, r} \\
& \text { and } R_{\{1\}, d}^{\min }>R_{\{1\}, r} \\
& \underline{\text { Case } 3 c}: R_{\{1\}, r}^{\min }<R_{\{1\}, d}, R_{\{2\}, r}^{\min }<R_{\{2\}, d} \\
& R_{\{2\}, d}^{\min }<R_{\{2\}, r}, \text { and } R_{\{1\}, d}^{\min }>R_{\{1\}, r} \\
& \text { Case }(1,3 a): R_{\mathcal{K}, r}<R_{\mathcal{K}, d} \text { and } R_{\{2\}, r}^{\min }<R_{\{2\}, d} \\
& \text { Case }(2,3 a): R_{\mathcal{K}, r}<R_{\mathcal{K}, d} \text { and } R_{\{1\}, r}^{\min }<R_{\{1\}, d} \\
& \underline{\text { Case }(1,3 b)}: R_{\mathcal{K}, r}>R_{\mathcal{K}, d} \text { and } R_{\{1\}, d}^{\min }<R_{\{1\}, r} \\
& \underline{\text { Case }(2,3 b)}: R_{\mathcal{K}, r}>R_{\mathcal{K}, d} \text { and } R_{\{2\}, d}^{\min }<R_{\{2\}, r} \\
& \text { Case }(1,3 c): R_{\mathcal{K}, r}=R_{\mathcal{K}, d}=R_{\{1\}, d}+R_{\{2\}, r} \\
& <R_{\{1\}, r}+R_{\{2\}, d} \\
& \underline{\text { Case }(2,3 c)}: R_{\mathcal{K}, r}=R_{\mathcal{K}, d}=R_{\{1\}, r}+R_{\{2\}, d} \\
& <R_{\{1\}, d}+R_{\{2\}, r}
\end{aligned}
$$

where in fading state $\underline{h},(40)-(50)$ are evaluated for $X_{k} \sim$ $\mathcal{C N}\left(0, P_{k}^{(x)}(\underline{h}) / \theta\right), k=1,2$, and $X_{r} \sim \mathcal{C N}\left(0, P_{r}^{(x)}(\underline{h}) / \bar{\theta}\right)$ for $x=i,(l, n)$.

The following theorem summarizes the form of $\underline{P}^{D F}$ and presents an algorithm to compute it.

Theorem 2: The optimal policy $\underline{P}^{D F}(\underline{H})$ maximizing the DF sum-rate of a two-user ergodic fading orthogonal MARC is obtained by computing $\underline{P}^{(i)}(\underline{H})$ and $\underline{P}^{(l, n)}(\underline{H})$ starting with the inactive cases 1 and 2 , followed by the active cases $3 a, 3 b$, and $3 c$, in that order, and finally the boundary cases $(l, n)$, in the order that cases $(l, 3 c)$ are the last to be optimized, until for some case the corresponding $\underline{P}^{(i)}(\underline{H})$ or $\underline{P}^{(l, n)}(\underline{H})$ satisfies the 
case conditions. The optimal $\underline{P}^{D F}(\underline{H})$ is given by the optimal $\underline{P}^{(i)}(\underline{H})$ or $\underline{P}^{(l, n)}(\underline{H})$ that satisfies its case conditions and falls into one of the following three categories:

Inactive Cases: The optimal policy for the two users is such that one user water-fills over its link to the relay while the other water-fills over its link to the destination. The optimal relay policy $P_{r}^{D F}(\underline{H})$ is water-filling over its direct link to the destination.

Cases $(3 a, 3 b, 3 c)$ : The optimal user policy $P_{k}^{D F}(\underline{H})$, for all $k \in \mathcal{K}$, is opportunistic water-filling over its link to the relay for case $3 a$ and to the destination for case $3 b$. For case $3 c, P_{k}^{D F}(\underline{H})$, for all $k \in \mathcal{K}$, takes an opportunistic nonwater-filling form and depends on the channel gains of user $k$ at both receivers. The optimal relay policy $P_{r}^{D F}(\underline{h})$ is water-filling over its direct link to the destination.

Boundary Cases: The optimal user policy $P_{k}^{D F}(\underline{H})$, for all $k \in \mathcal{K}$, takes an opportunistic nonwater-filling form. The optimal relay policy $P_{r}^{D F}(\underline{H})$ is water-filling over its direct link to the destination.

Proof: The closed form expressions for the optimal policies for each case are developed in the Appendix. The need for an order in evaluating $\underline{P}^{D F}(\underline{H})$ is due to the following reasons. From Lemma 1, for any polymatroids defined by the set functions $f_{1}$ and $f_{2}$, an inactive case results when

$$
f_{1}(\mathcal{S})+f_{2}(\mathcal{K} \backslash \mathcal{S})<\min \left(f_{1}(\mathcal{K}), f_{2}(\mathcal{K})\right) \quad \text { for a } \mathcal{S} \subset \mathcal{K} \text {. }
$$

Thus, the condition in (51) for the inactive cases by definition precludes an active case. For $K=2$, these conditions simplify to those in (40) and (41) for cases 1 and 2, respectively. Furthermore, the inactive cases are also mutually exclusive. The remaining (active and boundary) cases satisfy the conditions

$$
\begin{aligned}
\text { Cases } 3 a, 3 b, 3 c: \quad & f_{1}(\mathcal{S})+f_{2}(\mathcal{K} \backslash \mathcal{S}) \\
& >\min \left(f_{1}(\mathcal{K}), f_{2}(\mathcal{K})\right)
\end{aligned}
$$

Boundary Cases :

$$
\begin{array}{r}
f_{1}(\mathcal{S})+f_{2}(\mathcal{K} \backslash \mathcal{S})=\min \left(f_{1}(\mathcal{K}), \quad f_{2}(\mathcal{K})\right) \\
\text { for one } \mathcal{S}^{*} \subset \mathcal{K} \quad(53) \\
f_{1}(\mathcal{S})+f_{2}(\mathcal{K} \backslash \mathcal{S})>\min \left(f_{1}(\mathcal{K}), f_{2}(\mathcal{K})\right) \\
\text { for all } \mathcal{S}\left(\neq \mathcal{S}^{*}\right) \subset \mathcal{K} .
\end{array}
$$

For $K=2$, the condition in (52) simplifies to those in (42)-(44) for cases $3 a, 3 b$, and $3 c$, respectively, while that in (53) simplifies to those in (45)-(50) for the respective boundary cases. Additionally, for cases $3 a, 3 b$, and $3 c$, we also have the requirement that the sum-rate at the relay is less than, greater than, and equal to that at the destination, respectively. The conditions in (51) and (52) are mutually exclusive. On the other hand, the equality condition for a boundary case $(l, n)$, for all $l, n$, is subsumed in the optimization while the inequality condition is satisfied for all except one subset of users $\mathcal{S}^{*}$ for which the equality condition holds. This in turn implies that case $(l, n)$ has one less inequality condition than case $n$. Since case $3 c$ has no inequality conditions, neither do cases $(1,3 c)$ and $(2,3 c)$. Thus, the optimality of cases $(1,3 c)$ and $(2,3 c)$ can be determined only after eliminating the optimality of all others just as the optimality of case $3 c$ is determined after that of cases $3 a$ and $3 b$. The order of all other active and boundary cases can be chosen arbitrarily, and for ease of presentation, we simply assume that the search algorithm first verifies the optimality of $\underline{P}^{(1)}(\underline{H})$, failing which it verifies the optimality of $P^{(2)}(H)$, followed by $\underline{P}^{(3 a)}(\underline{H}), \underline{P}^{(3 b)}(\underline{H})$, and $\underline{P}^{(3 c)}(\underline{H})$, and finally verifies the optimality of the boundary cases in the order $(1,3 a),(2,3 a)$, $(1,3 b),(2,3 b),(1,3 c)$, and $(2,3 c)$. Note, however, that cases $(1,3 c)$ and $(2,3 c)$ are mutually exclusive due to cases 1 and 2 being disjoint. Thus, the optimal $P^{*}(H)$ is only achieved by a unique $\underline{P}^{(i)}(\underline{H})$ or $\underline{P}^{(l, n)}(\underline{H})$ depending on the policy that satisfies its case conditions.

Remark 6: The conditions for cases $3 a, 3 b$, and $3 c$ can also be redefined to include the negation of all the conditions for the other cases. This in turn eliminates the need for an order in computing the optimal policy; however, the number of conditions that need to be checked to verify whether the optimal policy satisfies the conditions for cases $3 a$ or $3 b$ or $3 c$ remain unchanged relative to the algorithm in Theorem 2.

We now summarize the optimal power policies at the sources and the relay for the different cases as follows.

Optimal Relay Policy: In the orthogonal model we consider, the relay transmits directly to the destination on a channel orthogonal to the source transmissions. Thus, the relay to destination link can be viewed as a fading point-to-point link. In fact, in all cases the optimal relay policy involves water-filling over the fading states analogous to a fading point to point link (see [27]). However, the exact solution, including scale factors, depends on the case considered. Thus, for case 1 , maximizing the sum rate results in the relay using its power $P_{r}(\underline{H})$ to forward only the message from user 1 in every fading state in which it transmits. Similarly, for case 2, the relay cooperates entirely with user 2 . For the active cases, $3 a$ and $3 b$, the sum-rate may be achieved by an infinite number of feasible points on one or both of the sum-rate planes; the optimal cooperative strategy at the relay will differ for each such point. Thus, for a corner point the relay transmits a message from only one of the users while for all noncorner points the relay transmits both messages. For the boundary cases, the water-filling solution at the relay is dependent on the Lagrangian parameter(s) introduced to satisfy the boundary conditions.

Optimal User Policies: As with the relay, the optimal policies for the two users depend on the case considered. For cases 1 and 2 , the optimal policies are water-filling solutions to that receiver at which it achieves a lower rate. In fact, the conditions for case 1 in (40) suggest a network geometry in which source 1 and the relay are physically proximal enough to form a cluster and source 2 and the destination form another cluster; and vice-versa for case 2 . For cases $3 a$ and $3 b$, the optimal policies at the two users maximize the two-user multiple-access sum-rate (see [25], [26]) achieved at the relay and destination, respectively, and thus, the optimal policy for each user involves water-filling over its fading states to that receiver. The solution also exploits the multi-user diversity to opportunistically schedule the users in each use of the channel. The optimal policies for case $3 c$ require the users to allocate power such that the sum-rates achieved at 
both the relay and the destination are the same. This constraint has the effect that it preserves the opportunistic scheduling since the sum-rate involves the multi-access sum-rate bounds at both receivers. However, the solutions are no longer water-filling due to the fact that the equality (boundary) condition results in the function $f_{k}^{(3 c)}$ being a weighted sum of the functions $f_{k}^{(3 a)}$ and $f_{k}^{(3 b)}$ for cases $3 a$ and $3 b$, respectively. The same observation holds true for the boundary cases too since $f_{k}^{(l, n)}$ are weighted sums of the functions for cases $l$ and $n$.

Remark 7: The case conditions in (40)-(50) require averaging over the channel states; thus, the case that maximizes the sum-rate depends on the average power constraints and the channel statistics (including network topology).

Remark 8: The optimal policy for each source for cases 1, $2,3 a$, and $3 b$ depends on the channel gains at only one of the receivers. However, the optimal policy for the boundary cases, including case $3 c$, depends on the instantaneous channel states at both receivers. Furthermore, all the cases exploiting the multiuser diversity require a centralized protocol to coordinate the opportunistic scheduling of users.

\section{V. $K$-USER GENERALIZATION AND DF RATE REGION}

\section{A. K-User Sum-Rate Analysis}

We use Lemma 1 to extend the two-user analysis in Section IV to $K$ users (and a fixed $\theta$ ). Recall that $\mathcal{R}_{D F}^{\theta}$ in Theorem 1 is given by a union of the intersection of polymatroids, where the union is over all power policies. From Lemma 1, we have that the maximal $K$-user sum-rate tuple is achieved by an intersection that either belongs to active set or to the inactive set. We index the $2^{K}-2$ nonempty subsets $\mathcal{S}^{(l)}$ of $\mathcal{K}$ via a superscript $l=1,2, \ldots, 2^{K}-2$. For a $K$-user MARC, there are $\left(2^{K}-2\right)$ possible intersections of the inactive kind with sum-rate $S^{(l)}$ given by

$$
\begin{gathered}
\text { case } l: S^{(l)}=R_{\mathcal{S}^{(l)}, r}+R_{\mathcal{K} \backslash \mathcal{S}^{(l)}, d} \text {, s.t. } R_{\mathcal{S}^{(l)}, r}<R_{\mathcal{S}^{(l)}, d}^{\min } \\
\text { and } R_{\mathcal{K} \backslash \mathcal{S}^{(l)}, d}<R_{\mathcal{K} \backslash \mathcal{S}^{(l)}, d}^{\min }, \quad l=1,2, \ldots, 2^{K}-2
\end{gathered}
$$

where $R_{\mathcal{A}, j}$ and $R_{\mathcal{A}, j}^{\min }$ are as defined in Section III and for $j=$ $r, d$, are given by the bounds (11) and (12), respectively. The sum-rates $S^{(i)}$, for the active cases $i=3 a, 3 b, 3 c$, are

$$
\begin{array}{ll}
\underline{\text { case } 3 a}: S^{(3 a)}=R_{\mathcal{K}, r} & \begin{array}{l}
\text { s.t. } R_{\mathcal{K}, r}<R_{\mathcal{K}, d} \\
\text { and } R_{\mathcal{K}, r}<S^{(l)} \text { for all } l
\end{array} \\
\underline{\text { case } 3 b}: S^{(3 b)}=R_{\mathcal{K}, d} & \begin{array}{l}
\text { s.t. } R_{\mathcal{K}, d}<R_{\mathcal{K}, r} \\
\text { and } R_{\mathcal{K}, d}<S^{(l)} \text { for all } l
\end{array} \\
\underline{\text { case } 3 c}: S^{(3 c)}=R_{\mathcal{K}, r} & \begin{array}{l}
\text { s.t. } R_{\mathcal{K}, d}=R_{\mathcal{K}, r}<S^{(l)} \\
\text { for all } l .
\end{array}
\end{array}
$$

Finally, the sum-rate $S^{(l, n)}$, for the boundary cases totaling $3\left(2^{K}-2\right)$ and enumerated as cases $(l, n), l=1,2, \ldots, 2^{K}-2$, $n=3 a, 3 b, 3 c$, are

$$
\begin{aligned}
& \underline{\operatorname{case}(l, 3 a)}: S^{(l, 3 a)}=R_{\mathcal{K}, r} \\
& \text { s.t. } R_{\mathcal{K}, r}=R_{\mathcal{S}^{(l)}, r}+R_{\mathcal{K} \backslash \mathcal{S}}{ }^{(l), d}=S^{(l)}<R_{\mathcal{K}, d} \\
& \operatorname{case}(l, 3 b): S^{(l, 3 b)}=R_{\mathcal{K}, d} \\
& \text { s.t. } R_{\mathcal{K}, d}=R_{\mathcal{S}^{(l)}, r}+R_{\mathcal{K} \backslash \mathcal{S}^{(l)}, d}=S^{(l)}<R_{\mathcal{K}, r} \\
& \underline{\operatorname{case}(l, 3 c)}: S^{(l, 3 c)}=R_{\mathcal{K}, r} \\
& \text { s.t. } R_{\mathcal{K}, r}=R_{\mathcal{K}, d}=R_{\mathcal{S}^{(l)}, r}+R_{\mathcal{K} \backslash \mathcal{S}(l), d}=S^{(l)}
\end{aligned}
$$

where the subset $\mathcal{S}$ is chosen to correspond to the appropriate case $l$.

Remark 9: The constraint for case $l$ in (54) are obtained directly from the requirement that the $K$-user sum-rate constraints at the two receivers are larger than that for case $l$ (see (51)).

The $K$-user sum-rate optimization problem for cases $i$ and $(l, n)$ can be written as

$$
\begin{gathered}
\max _{\underline{P}(\underline{H}) \in \mathcal{P}} S^{(i)} \text { or } \max _{\underline{P}(\underline{H}) \in \mathcal{P}} S^{(l, n)} \\
\text { s.t. } \mathbb{E}\left[P_{k}(\underline{H})\right] \leq \bar{P}_{k}, \\
P_{k}(\underline{h}) \geq 0, k=1,2, \ldots, K, r, \text { and for all } \underline{h} .
\end{gathered}
$$

An inactive case $l$ results when the conditions for that case in (54) are satisfied. The active cases $3 a, 3 b$, and $3 c$ result when the conditions in (55), (56), and (57) are satisfied, respectively. A boundary case results when the $K$-user sum-rate for case $n$ is equal to that for case $l$ as indicated in (58)-(60). Finally, as before, the achievable maximum sum-rate is given by (15) and (16) when $\theta$ can be varied. The DF sum-rate optimization problem here is analagous to the two-user case and in the interest of space, we simply summarize our results in the following theorem.

Theorem 3: The optimal power policy $\underline{P}^{*}(\underline{H})$ that maximizes the DF sum-rate of a $K$-user ergodic fading orthogonal Gaussian MARC is obtained by computing $\underline{P}^{(i)}(\underline{H})$ and $\underline{P}^{(l, n)}(\underline{H})$ starting with the inactive cases $1,2, \ldots, 2^{K}-2$, followed by the active cases $3 a, 3 b$, and $3 c$, and finally the boundary cases $(l, n)$, choosing cases $(l, 3 c)$ after computing $\underline{P}^{(l, n)}(\underline{H})$ for cases $(l, 3 a)$ and $(l, 3 b)$ for all $l$, until for some case the corresponding $\underline{P}^{(i)}(\underline{H})$ or $\underline{P}^{(l, n)}(\underline{H})$ satisfies the case conditions. The optimal $\underline{P}^{D F}(\underline{H})$ is given by the optimal $\underline{P}^{(i)}(\underline{H})$ or $\underline{P}^{(l, n)}(\underline{H})$ that satisfies its case conditions and falls into one of the following three categories:

Inactive Cases: The optimal user policy $P_{k}^{D F}(\underline{H})$, for all $k \in$ $\mathcal{K}$, is multi-user opportunistic water-filling over its bottle-neck (rate limiting) link to the relay among users in $\mathcal{S}$ or the destination among users in $\mathcal{K} \backslash \mathcal{S}$. The optimal relay policy $P_{r}^{D F}(\underline{H})$ is water-filling over its direct link to the destination.

Active Cases $(3 a, 3 b, 3 c)$ : The optimal user policy $P_{k}^{D F}(\underline{H})$, for all $k \in \mathcal{K}$, is opportunistic water-filling over its link to the relay for case $3 a$ and to the destination for case $3 b$. For case $3 c$, $P_{k}^{D F}(\underline{H})$, for all $k \in \mathcal{K}$, takes an opportunistic nonwater-filling form. The optimal relay policy $P_{r}^{D F}$ is water-filling over the relay-destination link. 
Boundary Cases: The optimal user policy $P_{k}^{D F}(\underline{H})$, for all $k \in \mathcal{K}$, takes an opportunistic nonwater-filling form. The optimal relay policy $P_{r}^{D F}(\underline{H})$ is water-filling over its direct link to the destination.

Based on the optimal DF policies, one can conclude that the topology of the network affects the form of the solution with the classic multi-user opportunistic water-filling solutions applicable only for the sources-relay or the relay-destination clustered models. For all other partially clustered or nonclustered networks, the solutions are a combination of single- and multi-user water-filling and nonwater-filling but opportunistic solutions.

\section{B. K-User Rate Region}

Analogously to the two-user analysis, one can also generalize the sum-rate analysis above to derive the optimal policies for all points on the boundary of the $K$-user DF rate region. For brevity, we outline the approach below.

We start with the observation that the DF rate region, $\mathcal{R}_{D F}$, is convex, and thus, every point on the boundary of $\mathcal{R}_{D F}$ is obtained by maximizing the weighted sum $\sum_{k \in \mathcal{K}} \mu_{k} R_{k}, \mu_{k}>0$ for all $k$. As noted earlier, each point on the boundary of $\mathcal{R}_{D F}$ is obtained by an intersection of two polymatroids for some $\underline{P}(\underline{H})$. Thus, analogously to the sum-rate analysis for $\mu_{k}=1$ for all $k$, for arbitrary $\underline{\mu}=\left(\mu_{1}, \mu_{2}, \ldots, \mu_{K}\right), \sum_{k \in \mathcal{K}} \mu_{k} R_{k}$, is maximized by either an inactive or an active case.

Since the maximum value of $\sum_{k \in \mathcal{K}} \mu_{k} R_{k}$ over a feasible bounded polyhedron is achieved at a vertex of the polyhedron, for any $\underline{P}(\underline{H})$, the $\left(R_{1}, R_{2}, \ldots, R_{K}\right)$-tuple maximizing $\sum_{k \in \mathcal{K}} \mu_{k} R_{k}$ is given by a vertex of an $\mathcal{R}_{r}(\underline{P}(\underline{H})) \cap \mathcal{R}_{d}(\underline{P}(\underline{H}))$ polyhedron at which $\sum_{k \in \mathcal{K}} \mu_{k} R_{k}$ is a tangent. For the $2^{K}-2$ inactive cases, the polymatroid intersections are polytopes with constraints on the multi-access rates of all users in $\mathcal{S}$ and $\mathcal{K} \backslash \mathcal{S}$ at the relay and destination, respectively. Since bounds on the multi-access rates of $l$ users result in a polymatroid with $l$ ! vertices, the intersection of the two orthogonal sum-rate planes will result in a polytope with $(|\mathcal{S}| !)(|\mathcal{K} \backslash \mathcal{S}| !)$ vertices of which an appropriate vertex will maximize $\sum_{k \in \mathcal{K}} \mu_{k} R_{k}$. Each of the $3\left(2^{K}-2\right)$ boundary cases are also characterized by an intersection with $(|\mathcal{S}| !)(|\mathcal{K} \backslash \mathcal{S}| !)$ vertices since these active cases are such that only one point on the sum-rate plane is included in the region of intersection. Finally, for cases $3 a, 3 b$, and $3 c$, the intersection of $K$-dimensional polymatroids results in a $K$-dimensional polyhedron.

In general, the intersection of two polymatroids is not a polymatroid, and thus, unlike the case with polymatroids, greedy algorithms do not maximize the weighted sum of rates. This in turn implies that closed form expressions are not in general possible and determining the optimal power policies requires convex programming techniques. We comment specifically on two cases of most interest.

Remark 10: For the special case in which the optimal policies for all $\mu$ are such that the bounds at the relay are smaller than the bounds at the destination for all $\mathcal{S}$, i.e., $\mathcal{R}_{r}^{\theta} \subset \mathcal{R}_{d}^{\theta}$, the optimal user policies for all $\mu$ are multi-user water-filling solutions developed in [26, II.C] with the relay as the receiver. Note that this condition implies that all possible subsets of users achieve better rates at the destination than at the relay. This can happen when either all users are clustered closer to the destination or when the relay has a relatively high SNR link to the destination sufficient enough to achieve rate gains for all users at the destination. This case is interesting only if the rates achieved thus are larger than the MAC sum-capacity (without relay).

Remark 11: Similarly, for the special case in which the optimal policies for all $\mu$ are such that $\mathcal{R}_{d}^{\theta} \subset \mathcal{R}_{r}^{\theta}$, the optimal user policies are multi-user water-filling solutions with the destination as the receiver. This case occurs when case $3 b$ holds for all points on the boundary of the DF rate region. This condition implies that all possible subsets of users achieve better rates at the relay than they do at the destination which in turn suggests a geometry in which all subsets of users are clustered closer to the relay than to the destination. The optimal relay policy in all cases is a water-filling solution over its link to the destination. In the following section we show that for this case DF achieves the capacity region.

\section{OUTER BOUNDS}

Thus far, we have focused on the DF achievable scheme. It is worthwhile to understand the conditions under which DF can achieve the sum-capacity, and if possible, the capacity region, for an ergodic fading Gaussian MARC. To this end, we develop outer bounds for this channel using cut-set bounds. Specifically, we obtain our outer bounds by specializing the known cut-set bounds developed in [10] for a $K$-user half-duplex discrete memoryless (d.m.) MARC to the Gaussian case. We summarize these half-duplex d.m. bounds summarized below. As with DF, we focus on the case in which the bandwidth parameter $\theta$ is fixed a priori, and thus, is not part of the optimization of the outer bound rate region. For the case in which $\theta$ can be varied, the rate region will be a union over regions, one for each feasible $\theta$.

Proposition 3: For the orthogonal MARC with a fixed $\theta$ the capacity region is contained in the union of the set of rate tuples $\left(R_{1}, R_{2}, \ldots, R_{K}\right)$ that satisfy

$$
\begin{array}{r}
R_{\mathcal{S}} \leq \min \left\{\theta I\left(X_{\mathcal{S}} ; Y_{r} Y_{d, 1} \mid X_{\mathcal{S}^{c}}, U\right), \theta I\left(X_{\mathcal{S}} ; Y_{d, 1} \mid X_{\mathcal{S}^{c}}, U\right)\right. \\
\left.+\bar{\theta} I\left(X_{r} ; Y_{d, 2} \mid U\right)\right\}, \quad \text { for all } \mathcal{S} \subseteq \mathcal{K}
\end{array}
$$

where the union is taken over all distributions that factor as

$$
p(u)\left(\prod_{k=1}^{K} p\left(x_{k} \mid u\right)\right) p\left(x_{r} \mid u\right) p\left(y_{r} y_{d, 1} \mid x_{\mathcal{K}}\right) p\left(y_{d, 2} \mid x_{r}\right) .
$$

Remark 12: The time-sharing random variable $U \in \mathcal{U}$ ensures that the region defined by (62) is convex. One can apply Caratheodory's theorem [38] to this $K$-dimensional convex region to bound the cardinality of $\mathcal{U}$ as $|\mathcal{U}| \leq K+1$. 
Following techniques similar to those for proving the converse for Gaussian MAC, we obtain

$$
\begin{aligned}
R_{\mathcal{S}} \leq \min & \left\{\theta \log \left|\boldsymbol{\Sigma}+\sum_{k \in \mathcal{S}} \mathbf{g}_{k} P_{k} / \theta\right|\right. \\
& \left.\theta C\left(\sum_{k \in \mathcal{S}}\left|h_{d, k}\right|^{2} P_{k} / \theta\right)+\bar{\theta} C\left(\left|h_{d, r}\right|^{2} P_{r} / \bar{\theta}\right)\right\}
\end{aligned}
$$

where $\Sigma$ is the covariance matrix of a noise vector $Z=\left[Z_{r}\right.$ $\left.Z_{d, 1}\right]^{T}$

$$
\mathbf{g}_{k}=\left[\begin{array}{ll}
h_{r, k} & h_{d, k}
\end{array}\right]^{T}\left[\begin{array}{ll}
h_{r, k}^{*} & h_{d, k}^{*}
\end{array}\right]
$$

$h_{(\cdot)}^{*}$ is the complex conjugate of $h_{(\cdot)}$, and a conditional entropy theorem [39] is used to show that Gaussian signals $X_{k} \sim$ $\mathcal{C} \mathcal{N}\left(0, P_{k} / \theta\right), k=1,2$, and $X_{r} \sim \mathcal{C} \mathcal{N}\left(0, P_{r} / \bar{\theta}\right)$ maximize the bounds in (62). Using the fact that the ergodic channel is a collection of parallel nonfading channels, one for each fading state instantiation, the capacity region of an ergodic fading orthogonal Gaussian MARC is as described in the following theorem.

Theorem 4: The capacity region $\mathcal{C}_{O-\mathrm{MARC}}^{\theta}$ of an ergodic fading orthogonal Gaussian MARC with a fixed bandwidth parameter $\theta$ is contained in

$$
\mathcal{R}_{O B}^{\theta}(\underline{\bar{P}})=\bigcup_{\underline{P}(\underline{H}) \in \mathcal{P}}\left\{\mathcal{R}_{1}^{\theta}(\underline{P}(\underline{H})) \cap \mathcal{R}_{2}^{\theta}(\underline{P}(\underline{H}))\right\}
$$

where, for all $\mathcal{S} \subseteq \mathcal{K}$, we have

$$
\begin{aligned}
\mathcal{R}_{1}^{\theta}(\underline{P}(\underline{H}))=\{ & \left(R_{1}, R_{2}\right): R_{\mathcal{S}} \leq \mathbb{E} \\
& \left.\times\left[\theta \log \left|I+\sum_{k \in \mathcal{S}} \mathbf{G}_{k} P_{k}(\underline{H}) / \theta\right|\right]\right\}
\end{aligned}
$$

where

$$
\mathbf{G}_{k}=\left[\begin{array}{ll}
H_{r, k} & H_{d, k}
\end{array}\right]^{T}\left[\begin{array}{ll}
H_{r, k}^{*} & H_{d, k}^{*}
\end{array}\right]
$$

and

$$
\begin{aligned}
\mathcal{R}_{2}^{\theta}(\underline{P}(\underline{H}))=\{ & \left(R_{1}, R_{2}\right): R_{\mathcal{S}} \\
\leq & \mathbb{E}\left[\theta C\left(\sum_{k \in \mathcal{S}}\left|H_{d, k}\right|^{2} P_{k}(\underline{H}) / \theta\right)\right. \\
& \left.\left.+\bar{\theta} C\left(\left|H_{d, r}\right|^{2} P_{r}(\underline{H}) / \bar{\theta}\right)\right]\right\} .
\end{aligned}
$$

Remark 13: Comparing outer bounds in (69) with the DF bounds in (12), we see that the bounds at the destination are the same in both cases. However, unlike the DF bound at only the relay in (11), the cutset bound in (67) is a single-input multipleoutput (SIMO) bound with single-antenna transmitters and with the relay and the destination acting as a multiantenna receiver.

The expressions in (67) and (69) are concave functions of $P_{k}(\underline{H})$, for all $k$, and thus, the region $\mathcal{R}_{O B}$ is convex. Thus, as in Theorem 1, the region $\mathcal{R}_{O B}$ in (66) is a union of the intersections of the regions $\mathcal{R}_{1}(\underline{P}(\underline{H}))$ and $\mathcal{R}_{2}(\underline{P}(\underline{H}))$, where the union is taken over all $\underline{P}(\underline{H}) \in \mathcal{P}$ and each point on the boundary of $\mathcal{R}_{D F}$ is obtained by maximizing the weighted sum $\mu_{1} R_{1}+\mu_{2} R_{2}$ over all $\underline{P}(\underline{H}) \in \mathcal{P}$, and for all $\mu_{1}>0, \mu_{2}>0$. In [40], it is shown that the rate polytopes satisfying the full-duplex cutset bounds are polymatroids. Since the polytopes in (67) and (69) are obtained from the full-duplex case for the special case of orthogonal signaling, one can verify in a straightforward manner using Definition 1 that these are polymatroids as well.

\section{A. Optimal Sum-Rate Policies and Sum-Capacity}

Since $\mathcal{R}_{O B}^{\theta}$ is obtained completely as a union of the intersection of polymatroids, one for each choice of power policy, Lemma 1 can be applied to explicitly characterize the outer bounds on the sum-rate. Thus, the maximum sum-rate tuple is achieved by an intersection that belongs to either the active set or to the inactive set such that there are $2^{K}-2$ inactive cases, cases $3 a, 3 b$, and $3 c$, and $3\left(2^{K}-2\right)$ boundary cases. The analysis here is analagous to the $K$-user DF case and the optimization for each case involves writing the Lagrangian and the KKT conditions. The optimal policy $\underline{P}^{(o b)}(\underline{H})$ satisfies the conditions for only one of the cases. Comparing these optimal policies with that for DF, we have the following capacity theorem.

Theorem 5: The sum-capacity of a $K$-user ergodic fading orthogonal Gaussian MARC is achieved by DF when the optimal policies $\underline{P}^{(o b)}(\underline{H})$ and $\underline{P}^{D F}(\underline{H})$ for the cutset and DF bounds, respectively, satisfy the conditions for case $3 b$ and for no other case.

Proof: The proof follows from comparing the sum-rate expressions for all cases for the inner and outer bounds, respectively. For all those cases in which the SIMO cut-set bound dominates the sum-rate, the cutset bounds do not match the DF bounds. On the other hand, when the optimal policies $\underline{P}^{(o b)}(\underline{H})$ and $\underline{P}^{D F}(\underline{H})$ satisfy the conditions for case $3 b$, the bound on $R_{\mathcal{K}}$ at the destination dominates for both the inner and the outer bounds. Furthermore, since this sum-rate bound at the destination is the same for both DF and the outer bounds, we have $\underline{P}^{(o b)}(\underline{H})=\underline{P}^{D F}(\underline{H})=\underline{P}^{(3 b)}(\underline{H})$, and thus, DF achieves the sum-capacity.

Remark 14: Recall that case $3 b$ corresponds to a clustered geometry in which the relay is clustered with all sources such that the cooperative multi-access link from the sources and the relay to the destination is the bottleneck link.

Remark 15: The set of power policies, $\mathcal{B}^{(i)}$ and $\mathcal{B}^{(l, n)}$, are defined by the appropriate conditions for the DF and outer bounds which are not necessarily the same (since the bounds are not exactly the same). However, when case $3 b$ maximizes both the inner and outer bounds, we have $\underline{P}^{D F}(\underline{H})=\underline{P}^{(o b)}(\underline{H})=$ $\underline{P}^{(3 b)}(\underline{H}) \in \mathcal{B}^{(3 b)}$ for both bounds.

\section{B. Capacity Region}

One can similarly write the rate expressions and the KKT conditions for every point on the boundary of $\mathcal{R}_{O B}^{\theta}$. The analysis is similar to that for the $K$-user orthogonal MARC under DF developed in Section V-B. From Theorem 4, every point 
$\sum_{k \in \mathcal{K}} \mu_{k} R_{k}$ on $\mathcal{R}_{O B}^{\theta}$ results from an intersection of two polymatroids. For those cases in which the intersection is an inactive case, both the SIMO cut-set bound at the relay and destination and the cooperative cut-set bound at the destination are involved, and thus, one cannot achieve capacity. This is also true for the boundary cases. For cases $3 a, 3 b$, and $3 c$, in which the polymatroid intersection also has $2^{K}-1$ constraints, and hence, $K$ ! corner points on the dominant $K$-user sum-rate face, $\sum_{k \in \mathcal{K}} \mu_{k} R_{k}$ is maximized by a corner point (vertex) of the resulting polytope. Any polytope that results from some or all of the SIMO bounds will be larger than the corresponding DF inner bounds. On the other hand, since $\mathcal{R}_{d}^{\theta}$ and $\mathcal{R}_{2}^{\theta}$, the DF and outer bound rate regions, respectively, at the destination are the same, the cut-set bounds can be tight when these bounds dominate the weighted rate sum. This occurs when the power policies maximizing the DF and outer outer bounds result in case $3 b$. Thus, for the cut-set bounds to be tight for a given $\underline{\mu}$ and $\theta$, we require

$$
\begin{aligned}
& \mathcal{R}_{2}^{\theta}\left(\underline{P}^{(o b)}(\underline{H}, \underline{\mu}, \theta)\right) \subset \mathcal{R}_{1}^{\theta}\left(\underline{P}^{(o b)}(\underline{H}, \underline{\mu}, \theta)\right), \quad \text { and } \\
& \mathcal{R}_{d}^{\theta}\left(\underline{P}^{D F}(\underline{H}, \underline{\mu}, \theta)\right) \subset \mathcal{R}_{r}^{\theta}\left(\underline{P}^{D F}(\underline{H}, \underline{\mu}, \theta)\right)
\end{aligned}
$$

where $\underline{P}^{(o b)}(\cdot)$ and $\underline{P}^{D F}(\cdot)$ denote the power policies maximizing $\sum_{k \in \mathcal{K}} \mu_{k} R_{k}$ for DF and the outer bounds, respectively, for a given $\theta$. Furthermore, when (70) is satisfied, we have $\underline{P}^{(o b)}(\cdot)=\underline{P}^{D F}(\cdot)=\underline{P}^{(3 b)}(\cdot)$, i.e., DF achieves the optimal weighted sum of rates $\sum_{k \in \mathcal{K}} \mu_{k} R_{k}$ for a given $\mu$. When this requirement holds for all $\mu$, we obtain the capacity region. This observation is summarized in the following theorem.

Theorem 6: The capacity region $\mathcal{C}_{O-\mathrm{MARC}}^{\theta}$ of an ergodic orthogonal Gaussian MARC with a fixed $\theta$ is achieved by DF when every point $\sum_{k \in \mathcal{K}} \mu_{k} R_{k}$ in the DF and the outer bound rate regions is achieved by case $3 b$ such that $\underline{P}^{(o b)}\left(\underline{H}, \mu_{1}, \mu_{2}, \theta\right)=\underline{P}^{D F}\left(\underline{H}, \mu_{1}, \mu_{2}, \theta\right) \equiv$ $\underline{P}^{*}\left(\underline{H}, \mu_{1}, \mu_{2}, \theta\right)$. The requirement that case $3 b$ maximizes every point on the boundary of both the DF and outer bound rate regions implies that for both bounds, $\mathcal{R}_{d}^{\theta}\left(\underline{P}^{*}(\cdot)\right) \subset \mathcal{R}_{r}^{\theta}\left(\underline{P}^{*}(\cdot)\right)$ and $\mathcal{R}_{2}^{\theta}\left(\underline{P}^{*}(\cdot)\right) \subset \mathcal{R}_{1}^{\theta}\left(\underline{P}^{*}(\cdot)\right)$ such that

$$
\mathcal{C}_{O-\mathrm{MARC}}^{\theta}=\mathcal{R}_{d}^{\theta}\left(\underline{P}^{*}(\cdot)\right)=\mathcal{R}_{2}^{\theta}\left(\underline{P}^{*}(\cdot)\right) .
$$

Remark 16: For the special case in which $\underline{H}$ has uniform phase fading and the channel state information is not known at the transmitters such that $P_{k}^{(o b)}(\underline{H})=P_{k}^{(3 b)}(\underline{H})=\bar{P}_{k}$, for all $k \in \mathcal{T}$, Theorem 6 yields the capacity region of an ergodic phase fading orthogonal Gaussian MARC as developed in [3, Theorem 9].

Remark 17: For the case of variable $\theta$, the capacity region is still achieved only when case $3 b$ maximizes all possible weighted sum of rates for both the DF and the outer bounds. However, now the rate regions result from a maximization over all feasible $\theta$. For the case of choosing the same sum-rate maximizing $\theta^{*}$ over all channel states, this optimization involves searching over the entire range of $\theta \in[0,1]$, because the equation that the optimal $\theta$ satisfies involves averaging rates over channel states, and has to be computed numerically. However,

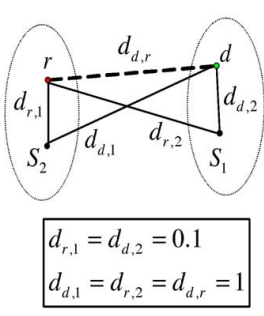

Topology 1



Topology 2
Fig. 6. Two-user MARCs with partially clustered (topology 1) and symmetric (topology 2) topologies.

for the case with $\theta$ varying with channel state, for each channel state, obtaining $\theta^{*}$ can be done more easily by solving an equation for the root instead of an exhaustive search (see, for e.g., [6] for $K=1$ ). For time-duplexed relay systems in [21], a quasi-concavity property of the sum-rate as a function of the time-sharing fraction is exploited to determine the sum-rate maximizing time fraction.

\section{ILLUSTRATION OF RESULTS}

We present numerical results for a two-user orthogonal MARC with Rayleigh fading links. We model the channel fading gains between receiver $m$ and transmitter $k$, for all $k$ and $m$, as

$$
H_{m, k}=\frac{A_{m, k}}{\sqrt{d_{m, k}^{\gamma}}}
$$

where $d_{m, k}$ is the distance between the transmitter and receiver, $\gamma$ is the path-loss exponent, and $A_{m, k}$ is a circularly symmetric complex Gaussian random variable with zero mean and unit variance such that $\left|H_{m, k}\right|^{2}$ is Rayleigh distributed with zero mean and variance $1 / d_{m, k}^{\gamma}$. We assume that $A_{m, k}$ are independent for all $m, k$. For the purpose of our illustration, we set the path-loss exponent $\gamma=3$. Finally, we use Monte Carlo methods to simulate the random parameters and evaluate the sum-rates for the inner DF and outer bounds.

Towards illustrating our results, we consider a two-user MARC under two different topologies as shown in Fig. 6. The first topology models a partially clustered MARC in which one of the users (user 1) is closer to the relay while the other (user 2) is closer to the destination than the other receiver. The second topology models a symmetric geometry where the users are equidistant from the destination and the relay. the average power levels in $\mathrm{dB}$ for topology 1 and 2 are $\bar{P}_{1}=\bar{P}_{2}=0 \mathrm{~dB}$, $\bar{P}_{r}=6 \mathrm{~dB}$ and $\bar{P}_{1}=\bar{P}_{2}=\bar{P}_{r}=4 \mathrm{~dB}$, respectively.

The partially clustered topology, referred to as topology 1 , in Fig. 6 models Case 1 in our analysis. For this topology, we plot the rate regions achieved at the relay and destination for three values of $\theta=0.25,0.5$, and 0.75 in Fig. 7. Also shown in each sub-plot (one for each $\theta$ ) is the MAC capacity region without resource allocation, i.e., assuming transmission at the same average power in each use of the channel. For all three choices of $\theta$, the optimal policies are those satisfying the conditions for case 1 such that users 1 and 2 waterfill over their bottleneck links to the destination and relay, respectively. However, as shown in Fig. 7, for all these choices of $\theta$, the DF sum-rate is strictly smaller than 

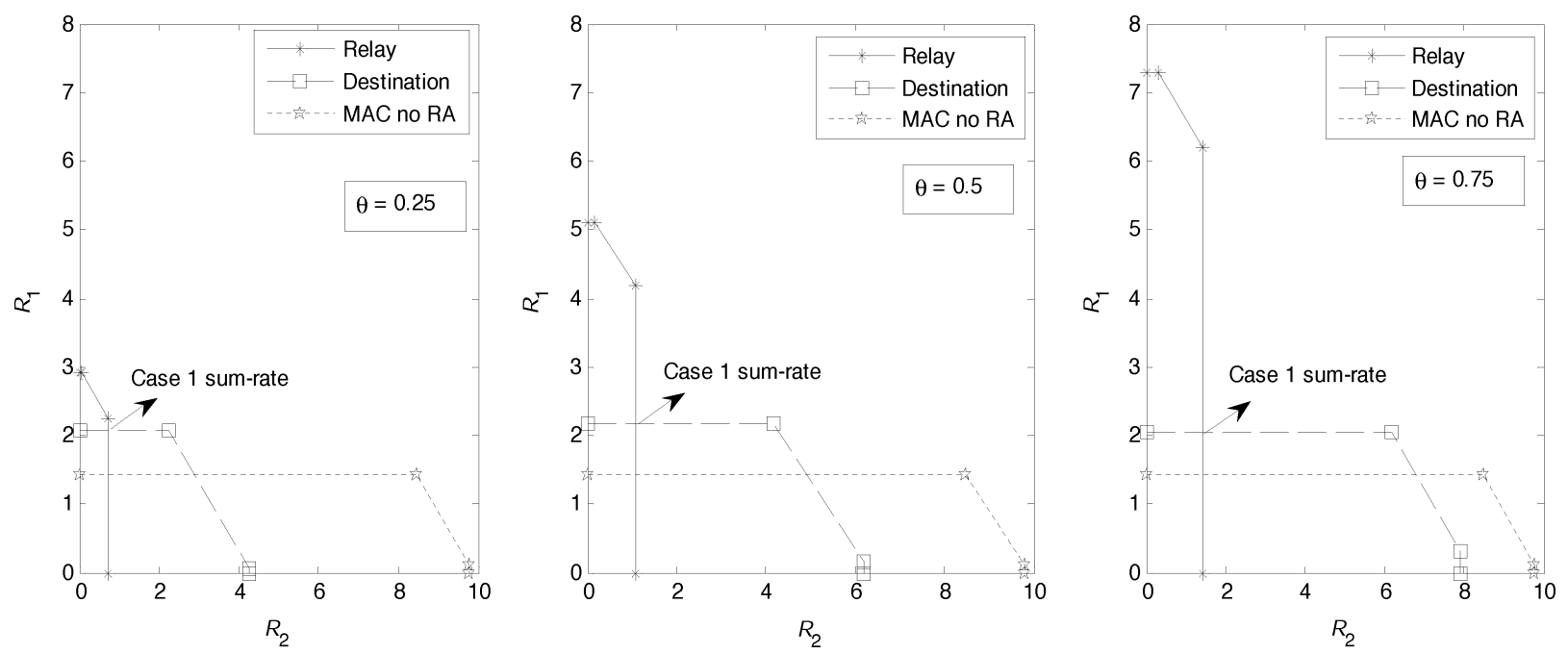

Fig. 7. Plot of the DF rate regions at the relay and destination and that of the MAC without resource allocation for $\theta=0.25,0.5$, and 0.8 .

the sum-rate of the ergodic fading Gaussian MAC (from sources to the destination) without resource allocation. This is due to two limitations: a) both users in the MARC are waterfilling to the distant receivers while in the MAC user 2 has the advantage of being closer to the intended receiver, and $b$ ) while the relay aids user 1 , the fractional bandwidth use resulting from the orthogonal half-duplex model significantly limits the rate gains. We make further comments on strategies for such geometries following the discussion of the second topology below.

Consider Topology 2 in Fig. 6. For the symmetric geometry modeled, in Fig. 8 we plot the inner (DF) and outer cutset bounds on the sum-rate for $\theta=1 / 2$ as a function of the relay position along the horizontal axis. As a result of the symmetric geometry, for every choice of the relay position, both the inner and outer bounds on the sum-rate are maximized by one of cases $3 a, 3 b$, or $3 c$. For each case, we use an iterative algorithm, as described in the Appendix, to compute the sum-rate maximizing user policies. For cases $3 a$ and $3 b$, the iterative algorithm simplifies to the iterative water-filling algorithm developed in [41] in which at each step the algorithm finds the single-user water-filling policy for each user while regarding the signals from the other user as noise. For case $3 c$, the optimal policy at each step is still obtained by regarding the signals from the other user as noise; however, the user policy at each step is no longer a water-filling solution. Finally, the optimality of DF when the sources are clustered relatively closer to the relay than to the destination is amply demonstrated in Fig. 8. The inner and outer bounds are also compared with the sum-capacity of the fading multi-access channel without a relay and $\theta=1$, shown by the dashed line that is a constant independent of the relay position. Also shown in Fig. 8 are the ranges of relay positions for cases $3 a, 3 b$, and $3 c$ for both DF and the cutset bounds.

For case $3 a$ in which the multiple access channel to the relay from the sources is the bottleneck link, for relay positions very close to the destination ( $>0.8$ in Fig. 8 ), the DF sum rate achieved for $\theta=1 / 2$ is strictly smaller than the waterfilling sum-capacity of the multiple access channel from the users to the destination (without the relay). A similar behavior is seen for the outer bound sum-rate when the relay is very proximal to the destination. This limitation is due to the orthogonal half-duplex constraint on the sources. One approach to increase the rate is to increase $\theta$ (as discussed below). The sum rate can also be potentially increased by allowing the sources to transmit in both orthogonal bands; however, it comes at a cost of increased complexity of analysis and solutions. In general, however, as has been established in earlier works (e.g., [3], [42]), a decode-and-forward relay provides significant rate and capacity benefits when it is physically closer to all the sources.

Finally, in Fig. 9 we illustrate the effect of three values of $\theta=0.4,0.5$, and 0.75 for Topology 2 . Relative to $\theta=1 / 2$, for $\theta>1 / 2$, the cooperative multiple-access link from the sources and relay to the destination (case $3 b$ ) remains a bottleneck link for a larger range of relay positions thereby increasing the range over which the sum capacity will be achieved. This is because for larger $\theta$, the rate achieved at the relay is larger. However, the smaller bandwidth allocated to the relay to destination link results in smaller case $3 b$ sum rates relative to the $\theta=0.4$ and the 0.5 curves. On the other hand, for relay positions closer to the destination, where the multiple access sum rate from the sources to the relay is the bottleneck link, the rates achieved are larger relative to the other two plots since $\theta$ is larger. The observations hold in reverse for $\theta=0.4$ relative to the larger values of $\theta$. The different choices of $\theta$ suggest that $\theta$ could be chosen a priori to maximize the rates achieved for a given network geometry. In all cases, as the relay approaches the destination, the performance of DF for this orthogonal signaling scheme we consider falls below that of the MAC sum-capacity.

Observations: The results illustrated thus far lead us to make the following observations:

1) The relay is most useful to all users only when all the users are clustered close to the relay such that the combined channel from the users and the relay to the destination is the bottleneck link.

2) Partially clustered geometries suffer from the limitation that users that are more distant from the relay than their intended receiver also need to be decoded by the relay. This in addition to the orthogonal half-duplex constraint limits the rates achieved significantly. One mitigating approach 


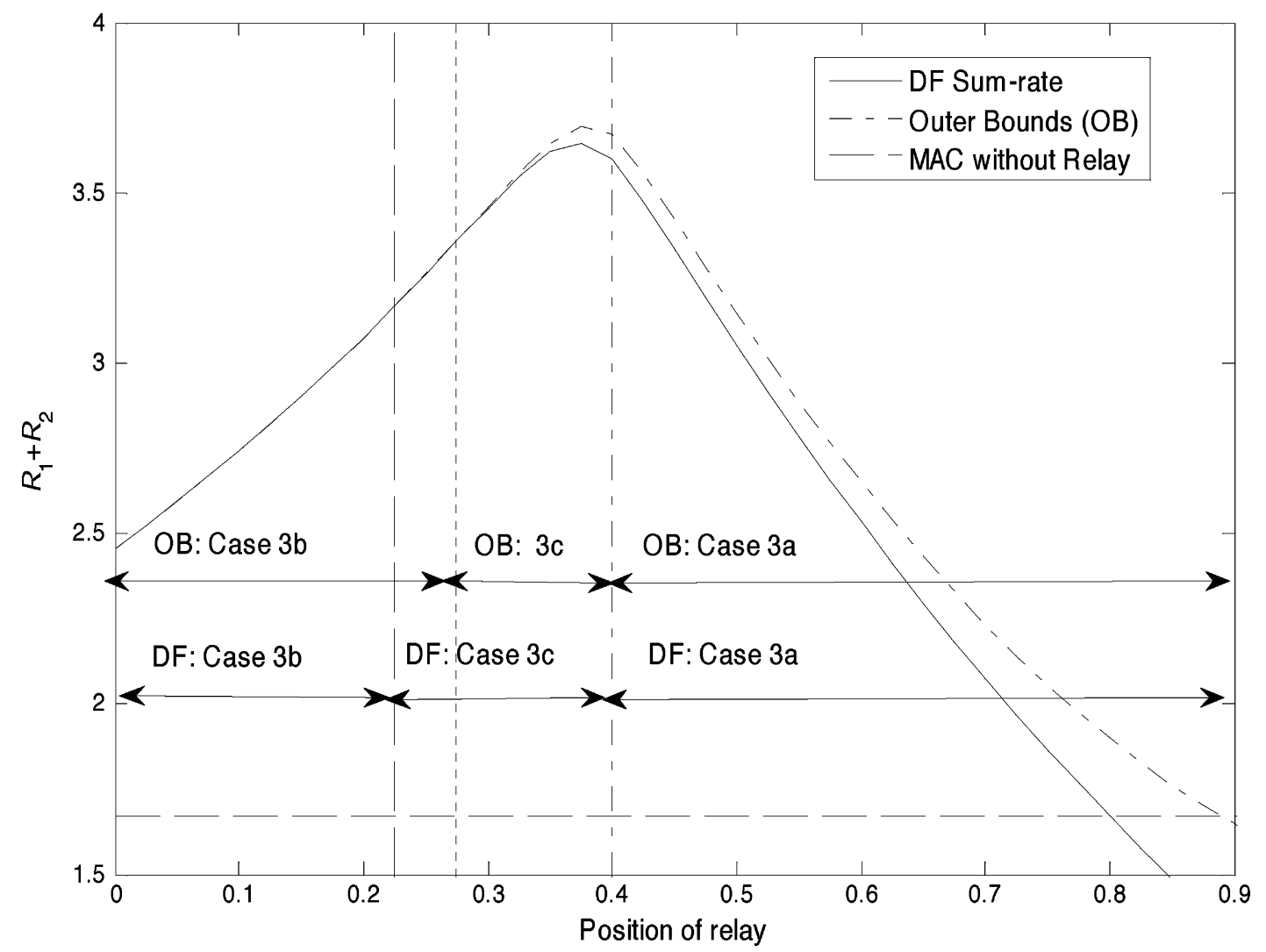

Fig. 8. Plot of inner and outer bounds on the sum-rate versus the relay position.

may be to use a combination of decoding and nondecoding strategies at the relay such as DF with compress-and-forward and amplify-and-forward. In these cases, optimizing the bandwidth parameter factor $\theta$ may also help. These may potentially enable better use of the relay resources albeit with increased complexity. Similar observations on combining strategies have also been made in [21] and [23].

3) Our results indicate that the channel model (orthogonal half-duplex model and choice of source-relay bandwidth fractions) as well as the network geometry determine the optimal solutions. In [21] and [23], the users and relay(s) are assumed to use orthogonal resources to avoid interference issues. We consider a model in which the users access the channel simultaneously over the same bandwidth but do not interfere with the relay. In all these models, the orthogonal use of bandwidth resources limits performance but allows practical implementation. However, the multiple-access nature of our model allows interference. In fact, while the sum-rate optimal DF power policies for our model involve opportunistic scheduling of users, which suggests scheduling users to transmit using orthogonal resources, our results clearly demonstrate the effect of the bandwidth fraction and the network geometry in determining the appropriate receiver to which to schedule all or a subset of users and highlight the subset of users for which the relay is most useful. These results can, therefore, potentially enable better user scheduling and bandwidth allocation in general multiple-access relay networks such as those studied in [21] and [23].
4) Effect of user and relay powers: While not explicitly shown in our illustration, we briefly remark on the effect of user and relay power on the DF sum-rate for a given geometry. First we note that for fixed user and relay average transmit powers, changing the value of $\theta$ is equivalent to changing the transmit powers in a specific way, i.e., increasing $\bar{P}_{k}$ by $1 / \theta, k=1,2$, and $\bar{P}_{r}$ by $1 / \bar{\theta}$. Our illustration demonstrates that for the range of $\theta$, and hence, the resulting small range of average node powers, and for the specific geometries considered, the optimizing case remains unchanged. In general, however, for a given geometry, the case maximizing the DF sum-rate will change when the transmit power of any node changes. For special cases such as symmetric or skewed geometries studied here, if the user powers scale proportionately (for fixed relay power and $\theta$ ), then the optimizing case will remain unchanged.

\section{CONCLUdING ReMARKS}

We have developed the maximum DF sum-rate and the sum-rate optimal power policies for an ergodic fading $K$-user half-duplex Gaussian MARC. The MARC is an example of a multiterminal network for which the multidimensionality of the policy set, the signal space, and the network topology space contribute to the complexity of developing capacity results resulting in few, if any, design rules for real-world communication networks. For a DF relay, the polymatroid intersection lemma we presented here allowed us to simplify the otherwise complicated analysis of developing the DF sum-rate optimal power policies for the two-user and $K$-user orthogonal MARC 


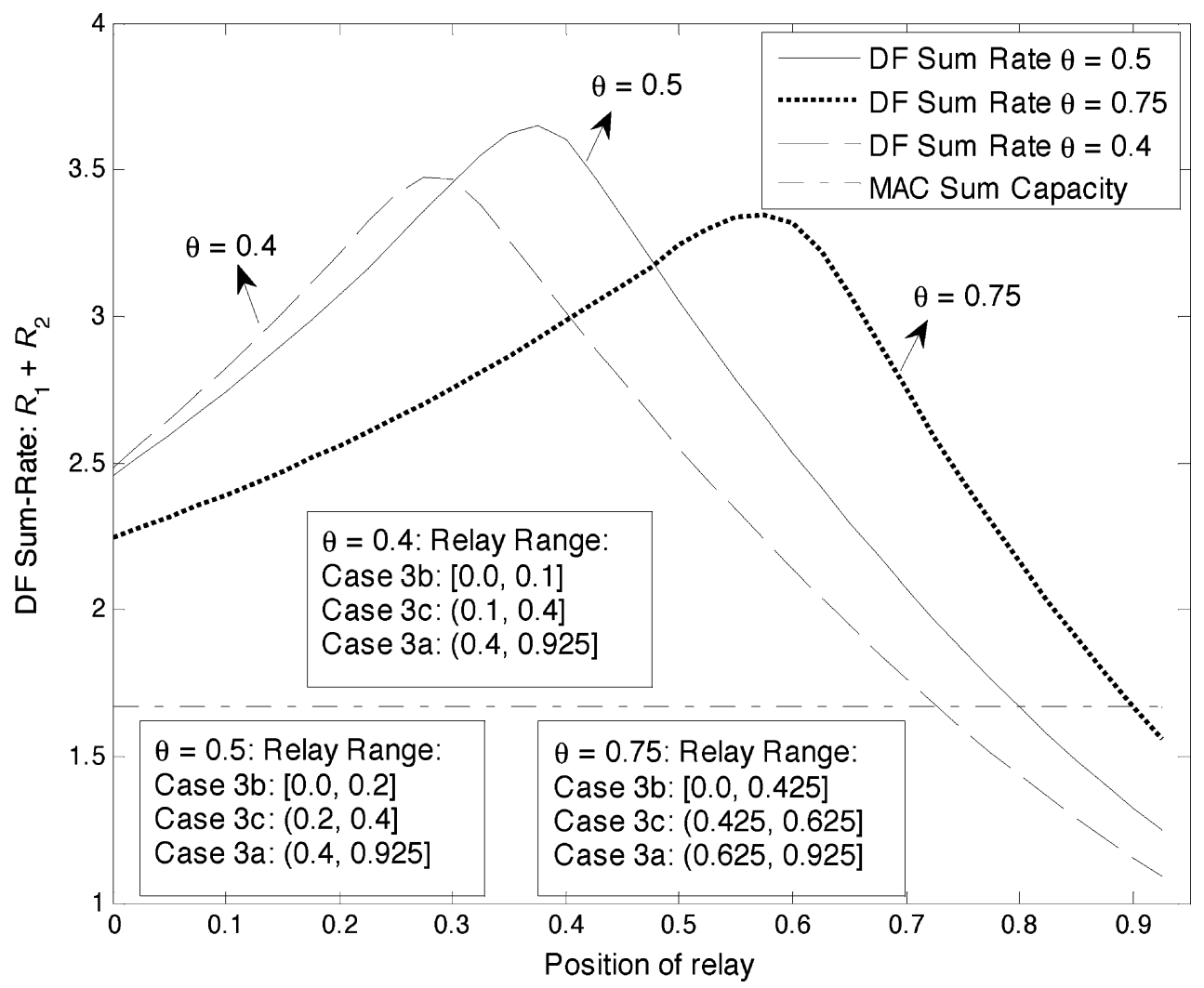

Fig. 9. DF sum rates achieved for $\theta=0.4,0.5$, and 0.75 for Topology 2 .

and the $K$-user outer bounds. The lemma allowed us to develop a broad topological classification of fading MARCs into one of following three types:

i) partially clustered MARCs in which a subset of all users form a cluster with the relay while the complementary subset of users form a cluster with the destination;

ii) clustered MARCs comprised of either sources-relay or relay-destination clustered networks;

iii) arbitrarily-clustered MARCs that are a combination of either the two clustered models or of a clustered and a partially clustered model.

The optimal policies for the inner DF and the outer cutset bounds for the orthogonal MARC model studied here lead to the following observations:

- DF achieves the sum-capacity of a class of source-relay clustered orthogonal MARCs for which the combined link from all sources and the relay to the destination, i.e., the link achieving the $K$-user sum-rate at the destination is the bottle-neck link. Furthermore, DF achieves the capacity region when for every weighted sum of user rates, the limiting bound is the weighted rate-sum achieved at the destination.

- For this sum-capacity achieving case, the optimal user policies for both the orthogonal and nonorthogonal MARCs are multi-user opportunistic water-filling solutions over their links to the destination and the optimal relay policy is a water-filling solution over its direct link to the destination.
- And for the remaining classes of MARCs, the optimal users policies are water-filling and nonwater-filling solutions for the partially clustered and arbitrarily clustered models, respectively.

For the partially clustered cases, we have shown that the optimal policy for each user is multi-user water-filling over its bottle-neck link to one of the receivers. Thus, the users that are clustered with the destination are forced to transmit at a lower rate to allow decoding of their signals at the relatively distant relay. Our results suggest that a useful practical strategy for the partially clustered topologies may be to allow those distant users that present little interference at the relay to communicate directly with the destination.

The optimal relaying strategy for all except the capacity achieving clustered case described above remains open. Given the complexity of finding the optimal signaling schemes for a given performance metric in multiterminal networks, a natural extension to this work could be to understand the gap in spectral efficiency between DF and the cutset outer bounds for fading MARCs using layered deterministic models. Such bounds have been developed recently for time-invariant interference channels and relay channels in [43] and [44], respectively, for fading Gaussian broadcast channels with no channel state information at the transmitter in [45], and for fading interference channels in [46] and [47].

A note on complexity: our theoretical analysis distinguishes between all possible polymatroid intersection cases in determining the optimal policy for a $K$-user system and, therefore, 
has a complexity that grows exponentially in the number of users. In practice, however, for two intersecting polymatroids the maximum of a weighted sum of rates and the optimizing policies can be computed using strongly polynomial-time algorithms [24, Theorem 47.4]. Finally, our analysis can also be extended to develop the DF regions for a more general nonorthogonal half-duplex model as well as the full-duplex MARC model. One can also study the ergodic rate regions for other relaying schemes studied for the MARC such as compress-and-forward and partial decode-and-forward [10], [48].

\section{APPENDIX \\ PROOF OF THEOREM 2}

The sum-rate maximizing DF power policy $\underline{P}^{*}(\underline{H})$ in Theorem 2 is obtained by sequentially determining the power policies $\underline{P}^{(i)}(\underline{H})$ and $\underline{P}^{(l, n)}(\underline{H})$ that maximize the sum-rate for cases $i$ and $(l, n)$, respectively, over all $\underline{P}(\underline{H}) \in \mathcal{P}$, until one of them satisfies the conditions for its case. The Lagragian maximizing the sum-rate for any case is given by

$$
\mathcal{L}^{(\cdot)}=S_{\theta}^{(\cdot)}-\sum_{k \in \mathcal{T}} \nu_{k} \mathbb{E}\left[P_{k}(\underline{H})-\bar{P}_{k}\right]+\sum_{k \in \mathcal{T}} \lambda_{k} P_{k}(\underline{H})
$$

where, for all $k \in \mathcal{T}, \nu_{k}$ are the dual variables associated with the power constraints in (5), $\lambda_{k} \geq 0$ are the dual variables associated with the positivity constraints $P_{k}(\underline{H}) \geq 0$, and $S_{\theta}^{(\cdot)}$ is given by (14) for each case. We now detail the optimization for each case separately starting with case 1 .

Case 1: The optimal policy is $\underline{P}^{(1)}(H)$ that maximizes (73) if it belongs to the open set $\mathcal{B}_{1}$ defined by the conditions

$$
\begin{aligned}
& R_{\{1\}, d}\left(\underline{P}^{(1)}(\underline{H})\right)<R_{\{1\}, r}^{\min }\left(\underline{P}^{(1)}(\underline{H})\right) \text { and } \\
& R_{\{2\}, r}\left(\underline{P}^{(1)}(\underline{H})\right)<R_{\{2\}, d}^{\min }\left(\underline{P}^{(1)}(\underline{H})\right)
\end{aligned}
$$

where

$$
\begin{aligned}
R_{1, r}^{\min }(\underline{P}(\underline{H})) & =\theta I\left(X_{1} ; Y_{r} \mid \underline{H}\right) \\
& =\mathbb{E}\left[\theta C\left(\frac{\left|H_{r, 1}\right|^{2} P_{1}(\underline{H}) / \theta}{1+\left|H_{r, 2}\right|^{2} P_{2}(\underline{H}) / \theta}\right)\right] \\
R_{2, d}^{\min }(\underline{P}(\underline{H})) & =\theta I\left(X_{2} ; Y_{d} \mid \underline{H}\right) \\
& =\mathbb{E}\left[\theta C\left(\frac{\left|H_{d, 2}\right|^{2} P_{2}(\underline{H}) / \theta}{1+\left|H_{d, 1}\right|^{2} P_{1}(\underline{H}) / \theta}\right)\right] .
\end{aligned}
$$

The KKT conditions for this case simplify to (17) with the superscript set to 1 . One can verify in a straightforward manner that these KKT conditions result in the power policies $P_{k}^{(1)}(\underline{h})$ and $P_{r}^{(1)}(\underline{h})$ given by (20) and (21), respectively.

Case 2: The optimal policy $\underline{P}^{(2)}(\underline{H})$ maximizes $(73)$ if it belongs to the open set $\mathcal{B}_{2}$ given by the conditions

$$
\begin{aligned}
& R_{\{1\}, r}(\underline{P}(\underline{H}))<R_{\{1\}, d}^{\min }(\underline{P}(\underline{H})) \quad \text { and } \\
& R_{\{2\}, d}(\underline{P}(\underline{H}))<R_{\{2\}, r}^{\min }(\underline{P}(\underline{H}))
\end{aligned}
$$

where $R_{\{2\}, r}^{\min }$ and $R_{\{1\}, d}^{\min }$ are given by (75) and (76), respectively, after replacing the user indices 1 by 2 and 2 by 1 . The optimal $P_{k}^{(2)}(\underline{H})$ and $P_{r}^{(2)}(\underline{H})$ are given by (20) and (21), respectively, with $(k, m)=(1, r),(2, d)$ provided $\underline{P}^{(2)}(\underline{H})$ satisfies (77)

Case 3: Consider the three cases $3 a, 3 b$, and $3 c$ shown in Fig. 3. Substituting the appropriate $S^{(i)}, i=3 a, 3 b, 3 c$, in (73), the sum-rate optimization for all three cases can be written as

$$
\max _{\underline{P}} \min \left(R_{\mathcal{K}, r}, R_{\mathcal{K}, d}\right)
$$

subject to average power and positivity constraints on $P_{k}$ for all $k$, where $R_{\mathcal{K}, j}$ denotes the sum-rate bound at receiver $j, j=d, r$. We write $\mathcal{B}_{3}$ to denote the open set consisting of all $\underline{P}(\underline{H}) \in \mathcal{P}$ that do not satisfy (74) and (77) either as strict inequalities or as a mixture of equalities and inequalities, where by a mixture we mean that a subset of the inequalities in (74) and (77) are satisfied with equality. We will later show that such sets of mixed equalities and inequalities in (74) and (77) corresponds to conditions for the various boundary cases (see also Figs. 4 and 5). Thus, $\underline{P}(\underline{H}) \in \mathcal{B}_{3}$ only when it does not satisfy the conditions for the inactive and the active-inactive boundary cases. By definition, $\mathcal{B}_{3}=\mathcal{B}_{3 a} \cup \mathcal{B}_{3 b} \cup \mathcal{B}_{3 c}$, where $\mathcal{B}_{i}, i=3 a, 3 b, 3 c$, is defined for case $i$ below.

The optimization in (78) is a multi-user generalization of the single-user max-min problem studied in [6] for the orthogonal single-user relay channel. The classical results on minimax optimization also apply to the multi-user sum-rate optimization in (78), and thus, the optimal policy $\underline{P}^{(i)}(\underline{H}), i=3 a, 3 b, 3 c$, satisfies one of following three conditions:

$$
\begin{aligned}
& \text { Case } 3 a:\left.\quad R_{\mathcal{K}, r}\right|_{\underline{P}^{(3 a)}(\underline{H})}<\left.R_{\mathcal{K}, d}\right|_{\underline{P}^{(3 a)}(\underline{H})} \\
& \left.R_{\{1\}, r}^{\min }\right|_{\underline{P}^{(3 a)}(\underline{H})}<\left.R_{\{1\}, d}\right|_{\underline{P}^{(3 a)}(\underline{H})} \\
& \left.R_{\{2\}, r}^{\min }\right|_{\underline{P}^{(3 a)}(\underline{H})}<\left.R_{\{2\}, d}\right|_{\underline{P}^{(3 a)}(\underline{H})} \\
& \text { Case } 3 b:\left.\quad R_{\mathcal{K}, r}\right|_{\underline{P}^{(3 b)}(\underline{H})}>\left.R_{\mathcal{K}, d}\right|_{\underline{P}^{(3 b)}}(\underline{H}) \\
& \left.R_{\{2\}, d}^{\min }\right|_{\underline{P}^{(3 b)}(\underline{H})}<\left.R_{\{2\}, r}\right|_{\underline{P}^{(3 b)}(\underline{H})} \\
& R_{\{1\}, d}^{\min _{\underline{P}^{(3 b)}(\underline{H})}}>\left.R_{\{1\}, r}\right|_{\underline{P}^{(3 b)}(\underline{H})} \\
& \text { Case } 3 c: \quad R_{\{2\}, d \underline{P}^{(3 c)}(\underline{H})}^{\min }<\left.R_{\{2\}, r}\right|_{\underline{P}^{(3 c)}(\underline{H})}
\end{aligned}
$$

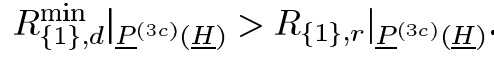

Note that the conditions in (79)-(81), evaluated at any $P \in \mathcal{B}_{3}$, are also conditions defining the sets $\mathcal{B}_{3 a}, \mathcal{B}_{3 b}$, and $\mathcal{B}_{3 c}$, respectively. We now present the optimal policies and sum-rates for each case in detail.

Case $3 a$ : For this case, from the KKT conditions in (17), $f^{(3 a)}$ in (23) depend only the sum-rate and channels gains of the two users at the relay. Thus, the problem simplifies to that for a $\mathrm{MAC}$ at the relay and the classical multi-user water-filling solution developed in [25] and [26] applies. The optimal user policies are thus given by (24), in which with the exception of the equality condition in (24), the optimal policies are unique, i.e., the optimal $P_{k}^{(3 a)}(\underline{H})$ at user $k$ in (24) is an opportunistic waterfilling solution that exploits the fading diversity in a multi-access channel from the sources to the relay. If the channel gains are continuously distributed, the equality condition occurs with probability 0 . Furthermore, even if the distributions were not continuous, one could choose to schedule one user or the other when the equality condition is met, thereby maintaining the opportunistic allocation policy. Finally, the optimal power policy 
at the relay is not explicitly obtained from the Lagrangian for this case as $S_{\theta}^{(3 a)}$ is the sum-rate achieved by the sources at the relay. However, since the sum-rate at the relay for this case is smaller than that at the destination, choosing the water-filling policy at the relay that maximizes the relay-destination link preserves the condition for this case, and thus, $P_{r}^{(3 a)}(\underline{H})$ is given by (21). When $P^{(3 a)}(\underline{H}) \in \mathcal{B}_{3}$, the requirement of satisfying (79), i.e., $\underline{P}^{(3 a)}(\underline{H}) \in \mathcal{B}_{3 a}$, simplifies to a threshold condition $\bar{P}_{r}>P_{u}\left(\bar{P}_{1}, \bar{P}_{2}\right)$ where $\bar{P}_{k}, k \in \mathcal{T}$, is defined in (5) and the threshold $P_{u}\left(\bar{P}_{1}, \bar{P}_{2}\right)$ is obtained by setting (79) to an equality. When $\underline{P}^{(3 a)}(\underline{H}) \in \mathcal{B}_{3}$ but $\underline{P}^{(3 a)}(\underline{H}) \notin \mathcal{B}_{3 a}, R_{1}+R_{2}$ is maximized by either case $3 b$ or case $3 c$. For $\underline{P}^{(3 a)}(\underline{H}) \notin \mathcal{B}_{3}$, as argued in Section IV, the sum-rate is not maximized by any $\underline{P}(\underline{H}) \in \mathcal{B}_{3}$.

Case $3 b$ : The optimal policy $P_{k}^{(3 b)}(\underline{H})$ at user $k$ for this case satisfies the KKT conditions in (17) with $f_{k}^{(i)}=f_{k}^{(3 b)}$. As with case $3 a$, here too, the optimal policy is an opportunistic water-filling solution and is given by (24) with the subscript ' $r$ ' changed to ' $d$ ' for all $k$ and with the superscript $i=3 b$. Further, for the relay node, the optimal $P_{r}^{(3 b)}(H)$ satisfies the KKT conditions in (17) for $f_{r}^{(3 b)}=f_{r}^{(1)}$, and is given by the water-filling solution in (21). Finally, for $\underline{P}^{(3 b)}(\underline{H}) \in \mathcal{B}_{3}$, the requirement $\underline{P}^{(3 b)}(\underline{H}) \in \mathcal{B}_{3 b}$ simplifies to satisfying the threshold condition $\bar{P}_{r}<P_{l}\left(\bar{P}_{1}, \bar{P}_{2}\right)$ where $P_{l}\left(\bar{P}_{1}, \bar{P}_{2}\right)$ is determined by setting (80) to an equality.

Case $3 c$ (equal-rate policy): The function $f_{k}^{(3 c)}$ is a weighted sum of $f_{k}^{(3 a)}$ and $f_{k}^{(3 b)}$ in which the Lagrange multiplier $\alpha$ accounts for the boundary condition in (81). Substituting $f_{k}^{(3 c)}$ in (17), we have the following KKT conditions:

$$
\begin{gathered}
\frac{\alpha\left|h_{r, k}\right|^{2}}{1+\sum_{k=1}^{2}\left|h_{r, k}\right|^{2} \frac{P_{k}(\underline{h})}{\theta}}+\frac{(1-\alpha)\left|h_{d, k}\right|^{2}}{1+\sum_{k=1}^{2}\left|h_{d, k}\right|^{2} \frac{P_{k}(\underline{h})}{\theta}} \\
\leq \nu_{k} \ln 2 \\
\text { with equality for } P_{k}(\underline{h})>0, k=1,2
\end{gathered}
$$

from which the optimal power policies simplify to (30). Determining the optimal $P_{k}^{(3 c)}(\underline{h}), k=1,2$, requires verifying each one of the three conditions in (30). Note that in contrast to cases $3 a$ and $3 b$, the opportunistic scheduling policy in (30) also depends on the user policies in addition to the channel states. Furthermore, the optimal solutions $P_{k}^{(3 c)}(\underline{H})$ do not take a water-filling form. Thus, for a given $P_{1}(\underline{h}), P_{2}(\underline{h})$ is given by

$$
P_{2}(\underline{h})=\text { positive root } x \text { of }(84) \text { if it exists, otherwise } 0
$$

where the root $x$ is determined by the following equation:

$$
\begin{gathered}
\frac{\alpha\left|h_{r, 2}\right|^{2}}{1+\left|h_{r, 1}\right|^{2} \frac{P_{1}(\underline{h})}{\theta}+\left|h_{r, 2}\right|^{2} \frac{x}{\theta}}+\frac{(1-\alpha)\left|h_{d, 2}\right|^{2}}{1+\left|h_{d, k}\right|^{2} \frac{P_{1}(\underline{h})}{\theta}+\left|h_{d, k}\right|^{2} \frac{x}{\theta}} \\
=\nu_{2} \ln 2 .
\end{gathered}
$$

Using $P_{2}(\underline{h})$ given by (84), $P_{1}(\underline{h})$ is obtained as the root of

$$
\begin{aligned}
& \frac{\alpha\left|h_{r, 1}\right|^{2}}{1+\left|h_{r, 1}\right|^{2} \frac{P_{1}(\underline{h})}{\theta}+\left|h_{r, 2}\right|^{2} \frac{P_{2}(\underline{h})}{\theta}} \\
& +\frac{(1-\alpha)\left|h_{d, 1}\right|^{2}}{1+\left|h_{d, k}\right|^{2} \frac{P_{1}(\underline{h})}{\theta}+\left|h_{d, k}\right|^{2} \frac{P_{2}(\underline{h})}{\theta}}=\nu_{1} \ln 2 .
\end{aligned}
$$

Thus, for all $\underline{h}$, starting with an initial $P_{1}(\underline{h})$, we iteratively obtain $P_{1}(\underline{h})$ and $P_{2}(\underline{h})$ until they converge to $P_{1}^{(3 c)}(\underline{h})$ and $P_{2}^{(3 c)}(\underline{h})$. The proof of convergence is detailed below. Finally, the optimal policies are determined over all $\alpha \in[0,1]$ to find an $\alpha^{*}$ that satisfies the equal rate condition in (81).

Proof of Convergence: The proof follows along the same lines as that detailed in [42, p. 3440] and relies on the fact that the maximizing function $S_{\theta}^{(3 c)}$ is a strictly concave function of $P_{1}(\underline{H})$ and $P_{2}(\underline{H})$ and is bounded from above because of the power constraints at the source and relay nodes. At each iteration, the optimal $P_{1}(\underline{H})$ and $P_{2}(\underline{H})$ are the KKT solutions that maximize the objective function. Thus, after each iteration, the objective function either increases or remains the same. It is easy to check that for a given $P_{1}(\underline{H})$ the objective function is a strictly concave function of $P_{2}(\underline{H})$, and thus, (84) yields a unique value of $P_{2}(\underline{H})$. Furthermore, the objective function is also a strictly concave function of $P_{1}(\underline{H})$ for a fixed $P_{2}(\underline{H})$. Thus, as the objective function converges, $\left(P_{1}(\underline{H}), P_{2}(\underline{H})\right)$ also converges. Finally, $P_{1}(\underline{H})$ and $P_{2}(\underline{H})$ converge to the solutions of the KKT conditions, which is sufficient for $\left(P_{1}(\underline{H}), P_{2}(\underline{H})\right)$ to be optimal since the objective function is concave over all $\underline{P}(\underline{H}) \in \mathcal{P}$.

Case 4: (Boundary Cases): Recall that we define the sets $B_{i}, i=1,2,3 a, 3 b, 3 c$, as open sets to ensure that an optimal $\underline{P}^{D F}$ maximizes the sum-rate for a case only if it satisfies the conditions for that case. Since an optimal policy can lie on the boundary of any two such cases, we also consider six additional cases each of which lies at the boundary of an inactive and an active case. These boundary cases result when the conditions for an inactive case $l, l=1,2$, and an active case $n, n=3 a, 3 b, 3 c$, are such that the sum-rate is the same for both cases. We consider each of the six boundary cases separately and develop the optimal $\underline{P}^{(l, n)}(\underline{H})$ for each case. The requirement that the optimal $\underline{P}^{(l, n)}(\underline{H})$ satisfies the condition $S_{\theta}^{(l)}=S_{\theta}^{(n)}$ for the boundary case $(l, n)$ simplifies to

$$
\begin{aligned}
& \text { case }(1,3 a): R_{\{1\}, d}+R_{\{2\}, r}=R_{\mathcal{K}, r}<R_{\mathcal{K}, d} \\
& \text { case }(1,3 b): R_{\{1\}, d}+R_{\{2\}, r}=R_{\mathcal{K}, d}<R_{\mathcal{K}, r} \\
& \text { case }(1,3 c): R_{\{1\}, d}+R_{\{2\}, r}=R_{\mathcal{K}, r}=R_{\mathcal{K}, d} \\
& \text { case }(2,3 a): R_{\{1\}, r}+R_{\{2\}, d}=R_{\mathcal{K}, r}<R_{\mathcal{K}, d} \\
& \text { case }(2,3 b): R_{\{1\}, r}+R_{\{2\}, d}=R_{\mathcal{K}, d}<R_{\mathcal{K}, r} \\
& \text { case }(2,3 c): R_{\{1\}, r}+R_{\{2\}, d}=R_{\mathcal{K}, d}=R_{\mathcal{K}, r}
\end{aligned}
$$

where the conditions in (86)-(91) are evaluated at the appropriate $\underline{P}^{(l, n)}(\underline{H})$. In addition, to ensure disjoint sets, from (53) we require that $\underline{P}^{(l, n)}(\underline{H})$ also satisfy $(45)-(50)$ which are the conditions defining the the sets $\mathcal{B}_{(1,3 a)}$ through $\mathcal{B}_{(2,3 c)}$, respectively. Using (45)-(50), we write the Lagrangian for all boundary cases except cases $(1,3 c)$ and $(2,3 c)$ as

$$
\begin{aligned}
& \mathcal{L}^{(l, n)}= \alpha S_{\theta}^{(l)}+(1-\alpha) S_{\theta}^{(n)}-\sum_{k \in \mathcal{T}} \nu_{k} \mathbb{E}\left[P_{k}(\underline{H})-\bar{P}_{k}\right] \\
&+\sum_{k \in \mathcal{T}} \lambda_{k} P_{k}(\underline{h}), \quad l=1,2, n=3 a, 3 b \\
& \lambda_{k} P_{k}(\underline{h}) \geq 0, \quad \text { for all } \underline{h} \text { and } k \in \mathcal{T}
\end{aligned}
$$


and the Lagrangian for cases $(1,3 c)$ and $(2,3 c)$ as

$$
\begin{array}{r}
\mathcal{L}^{(l, 3 c)}=\alpha_{1} S_{\theta}^{(l)}+\alpha_{2} S_{\theta}^{(3 a)}+\left(1-\alpha_{1}-\alpha_{2}\right) S_{\theta}^{(3 b)} \\
-\sum_{k \in \mathcal{T}} \nu_{k} \mathbb{E}\left[P_{k}(\underline{H})-\bar{P}_{k}\right]+\sum_{k \in \mathcal{T}} \lambda_{k} P_{k}(\underline{h}) \\
l=1,2
\end{array}
$$

$\lambda_{k} P_{k}(\underline{h}) \geq 0, \quad$ for all $\underline{h}$ and $k \in \mathcal{T}$

where $\nu_{k}$ and $\lambda_{k} \geq 0$ are dual variables associated with the average power and positivity constraints on $P_{k}$, respectively. The variable $\alpha$ is the dual variable associated with all boundary cases with a single boundary condition while $\alpha_{1}$ and $\alpha_{2}$ are the dual variables associated with cases $(1,3 c)$ and $(2,3 c)$. The resulting KKT conditions, one for each $P_{k}(\underline{h}), k=1,2, r$, are

$$
\begin{aligned}
& \text { Case }(l, n \neq 3 c): \frac{\partial \mathcal{L}^{(l, n)}}{\partial P_{k}(\underline{h})}=f_{k}^{(l, n)}=\alpha f_{k}^{(l)}+(1-\alpha) f_{k}^{(n)} \\
& \leq \nu_{k} \ln 2 \\
& \text { Case }(l, n=3 c): \frac{\partial \mathcal{L}^{(l, n)}}{\partial P_{k}(\underline{h})}=f_{k}^{(l, n)}=\alpha_{1} f_{k}^{(l)}+\alpha_{2} f_{k}^{(3 a)} \\
& \quad+\left(1-\alpha_{1}-\alpha_{2}\right) f_{k}^{(3 b)} \leq \nu_{k} \ln 2
\end{aligned}
$$

where $f_{k}^{(l)}$ and $f_{k}^{(n)}$ are as defined earlier for cases $l$ and $n$ and equality holds in (96) and (97) for $P_{k}(\underline{h})>0$, for all $\underline{h}$. We now present the optimal policies for each case separately. Case (1,3a): From (96), the KKT conditions for this case are

$$
\begin{aligned}
& f_{1}^{(1,3 a)}=\frac{\alpha\left|h_{d, 1}\right|^{2}}{1+\left|h_{d, 1}\right|^{2} P_{1}(\underline{h}) / \theta}+\frac{(1-\alpha)\left|h_{r, 1}\right|^{2}}{1+\sum_{j=1}^{2}\left|h_{r, j}\right|^{2} P_{j}(\underline{h}) / \theta} \\
& \leq \nu_{1} \ln 2, \text { with equality if } P_{1}(\underline{h})>0 \\
& f_{2}^{(1,3 a)}=\frac{\alpha\left|h_{r, 2}\right|^{2}}{1+\left|h_{r, 2}\right|^{2} P_{2}(\underline{h}) / \theta}+\frac{(1-\alpha)\left|h_{r, 2}\right|^{2}}{1+\sum_{j=1}^{2}\left|h_{r, j}\right|^{2} P_{j}(\underline{h}) / \theta} \\
& \leq \nu_{2} \ln 2, \text { with equality if } P_{2}(\underline{h})>0 \\
& f_{r}^{(1,3 a)}=\frac{\alpha\left|h_{d, r}\right|^{2}}{1+\left|h_{d, r}\right|^{2} P_{r}(\underline{h}) / \bar{\theta}} \leq \nu_{r} \ln 2 \\
& \text { with equality if } P_{r}(\underline{h})>0
\end{aligned}
$$

which results in (39). As in case $3 c$, the optimal policies take an opportunistic nonwater-filling form and in fact can be obtained by the iterative algorithm described for that case. Finally, from (100), the optimal $P_{r}^{(1,3 a)}(\underline{H})$ is given by (32).

Case $(1,3 b)$ : The analysis for this case mirrors that for case $(1,3 a)$ and the optimal user policies are opportunistic nonwater-filling solutions given by (39) with $f_{k}^{(3 a)}$ replaced by $f_{k}^{(3 b)}, k=1,2$. On the other hand in contrast to case $(1,3 a)$ where $f_{r}^{(3 a)}=0$, since both $f_{r}^{(1)}$ and $f_{r}^{(3 b)}$ are nonzero, the optimal relay policy $P_{r}^{(2,3 a)}=P_{r}^{(1)}$.
Case (1,3c): For this case, the KKT conditions in (97) involves a weighted sum of $f_{k}^{(l)}, f_{k}^{(3 a)}$, and $f_{k}^{(3 b)}$. Thus, for $k=1,2,(k, m)=(1, d),(2, r)$, we have the KKT conditions

$$
\begin{aligned}
f_{1}^{(1,3 c)} & =\alpha_{1} f_{1}^{(1)}+\alpha_{2} f_{1}^{(3 a)}+\left(1-\alpha_{1}-\alpha_{2}\right) f_{1}^{(3 b)} \\
& \leq \nu_{1} \ln 2, \text { with equality if } P_{1}(\underline{h})>0 \\
f_{2}^{(1,3 c)} & =\alpha_{1} f_{2}^{(1)}+\alpha_{2} f_{2}^{(3 a)}+(1-\beta)(1-\alpha) f_{2}^{(3 b)} \\
& \leq \nu_{2} \ln 2, \text { with equality if } P_{2}(\underline{h})>0 \\
f_{r}^{(1,3 c)} & =\left(1-\alpha_{2}\right)\left|h_{d, r}\right|^{2} / C\left(\left|h_{d, r}\right|^{2} P_{r} / \bar{\theta}\right) \\
& \leq \nu_{r} \ln 2, \text { with equality if } P_{r}(\underline{h})>0
\end{aligned}
$$

where $\alpha_{1}$ and $\alpha_{2}$ are the dual variables associated with the equalities $R_{\mathcal{K}, d}=R_{\{1\}, d}+R_{\{2\}, r}$ and $R_{\mathcal{K}, d}=R_{\mathcal{K}, r}$, respectively, in (88). From (101) and (102), one can verify that the optimal user policies are opportunistic nonwater-filling solutions given by (39) with the superscript $(1,3 a)$ replaced by $(1,3 c)$. Finally, $P_{r}^{(1,3 c)}(\underline{H})$ is given by the water-filling solution in (32) with $\alpha$ replaced by $\left(1-\alpha_{2}\right)$.

Case (2,3a): The optimal user policies for this case and the KKT conditions they satisfy are given by (98), (99), and (39) when $f_{k}^{(1)}$ is replaced by $f^{(2)}$, for all $k$, and $g_{k}^{(\cdot)}$ is superscripted by $(2,3 a)$. Thus, here too, the optimal user policies are opportunistic nonwater-filling solutions. The optimal relay policy $P_{r}^{(2,3 a)}(\underline{H})$ is the same as that obtained in case $(1,3 a)$.

Case $(2,3 b)$ : The optimal user policies $P_{k}^{(2,3 b)}(\underline{H}), k=1,2$, are again opportunistic nonwater-filling solutions and are given by (98), (99), and (39) when $f_{k}^{(1)}$ and $f_{k}^{(3 a)}$ are replaced by $f^{(2)}$ and $f^{(3 b)}$, respectively, for all $k$, and $g_{k}^{(\cdot)}$ is superscripted by $(2,3 b)$. The optimal relay policy $P_{r}^{(2,3 b)}(\underline{H})$ is the same as that for case $(1,3 b)$.

Case $(2,3 c)$ : The optimal policy vector $\underline{P}^{(2,3 c)}(\underline{H})$ is the same as that for case $(1,3 c)$ with $f_{k}^{(1)}$ is replaced by $f^{(2)}$, for all $k$, and with the superscript $(2,3 c)$.

\section{REFERENCES}

[1] A. Sendonaris, E. Erkip, and B. Aazhang, "User cooperation diversity-Part I: System description,” IEEE Trans. Commun., vol. 51, no. 11, pp. 1927-1938, Nov. 2003.

[2] J. N. Laneman, D. N. C. Tse, and G. Wornell, "Cooperative diversity in wireless networks: Efficient protocols and outage behavior," IEEE Trans. Inf. Theory, vol. 50, no. 12, pp. 3062-3080, Dec. 2004.

[3] G. Kramer, M. Gastpar, and P. Gupta, "Cooperative strategies and capacity theorems for relay networks," IEEE Trans. Inf. Theory, vol. 51, no. 9, pp. 3027-3063, Sep. 2005.

[4] K. Azarian, H. El Gamal, and P. Schniter, "On the achievable diversity-multiplexing tradeoff in half-duplex cooperative channels," IEEE Trans. Inf. Theory, vol. 51, no. 12, pp. 4152-4172, Dec. 2005.

[5] A. Host-Madsen and J. Zhang, "Capacity bounds and power allocation for the wireless relay channel," IEEE Trans. Inf. Theory, vol. 51, no. 6, pp. 2020-2040, Jun. 2005.

[6] Y. Liang and V. Veeravalli, "Resource allocation for wireless relay channels," presented at the 38th Annu. Asilomar Conf. Signals, Systems, and Computers, Pacific Grove, CA, Nov. 2004.

[7] L. Sankaranarayanan, G. Kramer, and N. B. Mandayam, "Cooperative diversity in wireless networks: A geometry-inclusive analysis," in Proc. 43rd Annu. Allerton Conf. Commun., Control, and Computing, Monticello, IL, Sep. 2005, pp. 1598-1607.

[8] G. Kramer and A. J. van Wijngaarden, "On the white Gaussian multiple-access relay channel," in Proc. IEEE Int. Symp. Information Theory, Sorrento, Italy, Jun. 2000, p. 40.

[9] T. Cover and A. El Gamal, "Capacity theorems for the relay channel," IEEE Trans. Inf. Theory, vol. 25, no. 5, pp. 572-584, Sep. 1979. 
[10] L. Sankaranarayanan, G. Kramer, and N. B. Mandayam, "Hierarchical sensor networks: Capacity theorems and cooperative strategies using the multiple-access relay channel model," in Proc. 1st IEEE Conf. Sensor and Ad Hoc Communications and Networks, Santa Clara, CA, Oct. 2004, pp. 191-199.

[11] A. El Gamal and S. Zahedi, "Capacity of relay channels with orthogonal components," IEEE Trans. Inf. Theory, vol. 51, no. 5, pp. 1815-1817, May 2005.

[12] M. Yuksel and E. Erkip, "Multi-antenna cooperative wireless systems: A diversity-multiplexing tradeoff perspective," IEEE Trans. Inf. Theory, vol. 53, no. 10, pp. 3371-3393, Oct. 2007.

[13] D. Gündüz and E. Erkip, "Opportunistic cooperation by dynamic resource alloation," IEEE Trans. Wireless Commun., vol. 6, no. 4, pp. 1446-1454, Apr. 2007.

[14] A. S. Avestimehr and D. N. C. Tse, "Outage capacity of the fading relay channel in the low SNR regime," IEEE Trans. Inf. Theory, vol. 53, no. 4, pp. 1401-1415, Apr. 2007.

[15] L. Sankar, G. Kramer, and N. B. Mandayam, "User vs. relay cooperation in time-duplexed multi-access relay networks," Aug. 2008, arxiv.org e-print 0809.2226.

[16] R. Liu, J. Luo, and P. Spasojevic, "Adaptive transmission with variable-rate turbo bit-interleaved coded modulation," IEEE Trans. Wireless Commun., vol. 6, no. 11, pp. 3926-2936, Nov. 2007.

[17] A. Stefanov and E. Erkip, "Cooperative coding for wireless networks," IEEE Trans. Commun., vol. 52, no. 8, pp. 3926-2936, Sep. 2004.

[18] I. Maric and R. Yates, "Forwarding strategies for Gaussian parallelrelay networks," in Proc. IEEE Int. Symp. Inform. Theory, Chicago, IL, Jun. 2004, p. 269.

[19] I. Maric and R. Yates, "Bandwidth and power allocation for cooperative strategies in Gaussian relay networks," in Proc. 38th Annu. Asilomar Conf. Signals, Systems, and Computers, Pacific Grove, CA, Nov. 2004, pp. 1907-1911.

[20] Y. Yao, X. Cai, and G. B. Giannakis, "On energy efficiency and optimum resource allocation of relay transmissions in the low-power regime," IEEE Trans. Wireless Commun., vol. 4, no. 6, pp. 2917-2927, Nov. 2005.

[21] W. Mesbah and T. N. Davidson, "Power and resource allocation for orthogonal multiple access relay systems," EURASIP J. Adv. Sig. Proc., vol. 2008, no. 476125, p. 15, 2008.

[22] O. Oyman, "Opportunism in multiuser relay channels: Scheduling, routing, and spectrum reuse," in Proc. IEEE Int. Symp. Information Theory, Nice, France, Jun. 2007, pp. 286-290.

[23] S. Serbetli and A. Yener, "Relay assisted F/TDMA ad hoc networks: Node classification, power allocation, and relaying strategies," IEEE Trans. Commun., vol. 56, no. 6, pp. 937-947, June 2008.

[24] A. Schrijver, Combinatorial Optimization: Polyhedra and Efficiency. New York: Springer-Verlag, 2003.

[25] R. Knopp and P. Humblet, "Information capacity and power control in single-cell multiuser communications," in Proc. IEEE Int. Conf. Commun., Seattle, WA, Jun. 1995, pp. 331-335.

[26] D. N. C. Tse and S. V. Hanly, "Multiaccess fading channels-Part I: Polymatroid structure, optimal resource allocation and throughput capacities," IEEE Trans. Inf. Theory, vol. 44, no. 7, pp. 2796-2815, Nov. 1998.

[27] A. Goldsmith and P. Varaiya, "Capacity of fading channels with channel side information," IEEE Trans. Inf. Theory, vol. 43, no. 6, pp. 1986-1992, Nov. 1997.

[28] N. Jindal and A. Goldsmith, "Optimal power allocation for parallel broadcast channels with independent and common information," in Proc. IEEE Int. Symp. Inform. Theory, Chicago, IL, Jun. 2004, p. 215.

[29] L. Sankar, X. Shang, E. Erkip, and H. V. Poor, "Ergodic two-user interference channels: Sum-capacity and separability," IEEE Trans. Inf. Theory, to be published.

[30] S. Jafar, "The ergodic capacity of interference networks," Feb. 2009, arxiv.org e-print 0902.0838.

[31] R. G. Gallager, Information Theory and Reliable Communication. Hoboken, NJ: Wiley, 1968.

[32] T. M. Cover and J. A. Thomas, Elements of Information Theory. New York: Wiley, 1991.

[33] T. S. Han, "The capacity region of general multiple-access channel with certain correlated sources," Inf. Control, vol. 40, no. 1, pp. 37-60, Jan. 1979.

[34] L. Sankar, G. Kramer, and N. B. Mandayam, "Offset encoding for multiaccess relay channels," IEEE Trans. Inf. Theory, vol. 53, no. 10, pp. $1-8$, Oct. 2007.
[35] L. Sankaranarayanan, G. Kramer, and N. B. Mandayam, "Hierarchical wireless networks: Capacity theorems using the constrained multiple-access relay channel model," in Proc. 38th Annu. Asilomar Conf. Signals, Systems, and Computers, Pacific Grove, CA, Nov. 2004, pp. 1912-1916, invited paper.

[36] G. Kramer, "Models and theory for relay channels with receive constraints," in Proc. 42nd Annu. Allerton Conf. on Commun., Control, and Computing, Monticello, IL, Sep. 2004, pp. 1312-1321.

[37] S. Boyd and L. Vandenberghe, Convex Optimization. Cambridge, U.K.: Cambridge Univ. Press, 2004.

[38] H. G. Eggleston, Convexity. Cambridge, U.K.: Cambridge Univ. Press, 1958.

[39] J. A. Thomas, "Feedback can at most double Gaussian multiple access channel capacity," IEEE Trans. Inf. Theory, vol. 33, no. 5, pp. 711-716, Sep. 1987.

[40] L. Sankar, N. B. Mandayam, and H. V. Poor, "On the sum-capacity of degraded Gaussian multiaccess relay channels," IEEE Trans. Inf. Theory, vol. 55, no. 12, pp. 5394-5411, Dec. 2009.

[41] W. Yu and W. Rhee, "Iterative water-filling for Gaussian vector multiple-access channels," IEEE Trans. Inf. Theory, vol. 50, no. 1, pp. 145-152, Jan. 2004.

[42] Y. Liang, V. Veeravalli, and H. V. Poor, "Resource allocation for wireless fading relay channels: Max-min solution," IEEE Trans. Inf. Theory, vol. 53, no. 10, pp. 3432-3453, Oct. 2007.

[43] G. Bresler and D. N. C. Tse, "The two-user Gaussian interference channel: A deterministic view," Euro. Trans. Telecomm., vol. 19, no. 4, pp. 333-354, June 2008.

[44] S. Avestimehr, S. Diggavi, and D. N. C. Tse, "Wireless network information flow: A deterministic approach," Oct. 2007, arxiv.org e-print 0710.3781 .

[45] D. Tse, R. Yates, and Z. Li, "Fading broadcast channels with state information at the receivers," in Proc. 46th Annu. Allerton Conf. Commun., Control, and Computing, Monticello, IL, Sep. 2008, pp. 221-227.

[46] V. Aggarwal, L. Sankar, A. R. Calderbank, and H. V. Poor, "Ergodic layered erasure one-sided interference channels," in Proc. IEEE Information Theory Workshop, Taormina, Sicily, Italy, Oct. 2009, pp. 574-578.

[47] Y. Zhu and D. Guo, "Ergodic fading one-sided interference channels without state information at the transmitters," Nov. 2009, arxiv.org e-print 0911.1082.

[48] L. Sankaranarayanan, G. Kramer, and N. B. Mandayam, "Capacity theorems for the multiple-access relay channel," in Proc. 42nd Annu. Allerton Conf. on Commun., Control, and Computing, Monticello, IL, Sep. 2004, pp. 1782-1791.

Lalitha Sankar (S'92-A'95-M'07) received the B.Tech. degree from the Indian Institute of Technology, Bombay, the M.S. degree from the University of Maryland, College Park, and the Ph.D. degree from Rutgers University, Piscataway, NJ, in 2007.

Prior to her doctoral studies, she was a Senior Member of Technical Staff at AT\&T Shannon Laboratories. She is currently a Research Scholar at Princeton University, Princeton, NJ. Her research interests include wireless communications, network information theory, and information privacy, and secrecy.

Dr. Sankar was a recipient of a Science and Technology Postdoctoral Fellowship from the Council on Science and Technology at Princeton University during 2007-2010. For her doctoral work, she received the 2007-2008 Electrical Engineering Academic Achievement Award from Rutgers University.

Yingbin Liang (S'01-M'05) received the Ph.D. degree in electrical engineering from the University of Illinois at Urbana-Champaign (UIUC), Urbana, in 2005.

In 2005-2007, she was a postdoctoral research associate at Princeton University, Princeton, NJ. In 2008-2009, she was an Assistant Professor in the Department of Electrical Engineering, University of Hawaii, Honolulu. Since December 2009, she has been an Assistant Professor with the Department of Electrical Engineering and Computer Science, Syracuse University, Syracuse, NY. Her research interests include communications, wireless networks, and information theory.

Dr. Liang was a Vodafone Fellow at the UIUC during 2003-2005 and received the Vodafone-U.S. Foundation Fellows Initiative Research Merit Award in 2005. She also received the M. E. Van Valkenburg Graduate Research Award from the ECE Department, UIUC, in 2005. In 2009, she received the National Science Foundation CAREER Award, and the State of Hawaii Governor Innovation Award. 
Narayan B. Mandayam (S'90-M'95-SM'00-F'09) received the B.Tech. (Hons.) degree in 1989 from the Indian Institute of Technology, Kharagpur, and the M.S. and Ph.D. degrees in 1991 and 1994 from Rice University, Houston, TX, all in electrical engineering.

From 1994 to 1996, he was a Research Associate at the Wireless Information Network Laboratory (WINLAB), Department of Electrical and Computer Engineering, Rutgers University, Piscataway, NJ. In September 1996, he joined the faculty of the ECE Department, Rutgers, where he became Associate Professor in 2001 and Professor in 2003. Currently, he also serves as Associate Director at WINLAB. He was a visiting faculty fellow in the Department of Electrical Engineering, Princeton University, in Fall 2002, and a visiting faculty at the Indian Institute of Science in Spring 2003. His research interests are in various aspects of wireless data transmission with emphasis on techniques for cognitive radio networks including their implications for spectrum policy. Using constructs from game theory, communications, and networking, his work focusses on system modeling and performance, signal processing as well as radio resource management for enabling wireless technologies to support various applications.

Dr. Mandayam is a recipient of the Fred W. Ellersick Prize from the IEEE Communications Society in 2009 along with O. Ileri for their work on dynamic spectrum access models and spectrum policy. He is also the recipient of the Institute Silver Medal from the Indian Institute of Technology, Kharagpur, in 1989 and the National Science Foundation CAREER Award in 1998. He was selected by the National Academy of Engineering in 1999 for the Annual Symposium on Frontiers of Engineering. He is a coauthor with C. Comaniciu and H. V. Poor of the book Wireless Networks: Multiuser Detection in Cross-Layer Design (Springer). He has served as an Editor for the IEEE COMMUNICATION LETTERS (1999-2002) and the IEEE TRANSACTIONS ON WIRELESS COMMUNICATIONS (2002-2004). He has served as a guest editor of the IEEE JSAC Special Issues on Adaptive, Spectrum Agile, and Cognitive Radio Networks (2007) and Game Theory in Communication Systems (2008).
H. Vincent Poor (S'72-M'77-SM'82-F'87) received the Ph.D. degree in electrical engineering and computer science from Princeton University, Princeton, $\mathrm{NJ}$, in 1977.

From 1977 until 1990, he was on the faculty of the University of Illinois at Urbana-Champaign, Urbana. Since 1990, he has been on the faculty at Princeton, where he is the Dean of Engineering and Applied Science and the Michael Henry Strater University Professor of Electrical Engineering. His research interests are in the areas of stochastic analysis, statistical signal processing and information theory, and their applications in wireless networks and related fields. Among his publications in these areas are Quickest Detection (Cambridge University Press, 2009), co-authored with O. Hadjiliadis, and Information Theoretic Security (Now Publishers, 2009), co-authored with Y. Liang and S. Shamai.

Dr. Poor is a member of the National Academy of Engineering, a Fellow of the American Academy of Arts and Sciences, and an International Fellow of the Royal Academy of Engineering (U.K.). He is also a Fellow of the Institute of Mathematical Statistics, the Optical Society of America, and other organizations. In 1990, he served as President of the IEEE Information Theory Society, from 2004-2007 as the Editor-in-Chief of the IEEE TRANSACTIONS ON INFORMATION THEORY, and in 2009 as General Co-chair of the IEEE International Symposium on Information Theory, held in Seoul, South Korea. He received a Guggenheim Fellowship in 2002 and the IEEE Education Medal in 2005. Recent recognition of his work includes the 2008 Aaron D. Wyner Distinguished Service Award of the IEEE Information Theory Society, the 2009 Edwin Howard Armstrong Achievement Award of the IEEE Communications Society, the 2010 IET Ambrose Fleming Medal for Achievement in Communications, and the 2011 IEEE Eric E. Sumner Award. 